\title{
Taposiris Magna et Plinthine (2019)
}

Bérangère Redon, Sylvain Dhennin, Claire Somaglino, Matthieu Vanpeene, Lorenzo Medini, Louis Dautais, Séverine Marchi, Rim Saleh, Joachim Le Bomin, Julie Marchand, Charlène Bouchaud, Mennat-Allah El Dorry, Clémence Pagnoux, Robert Ryndziewicz, Tomasz Herbich, Maël Crépy, Alexandre Rabot, Paul François, Clementina Caputo, Mikaël Pesenti, Mahmoud Samir et Thomas Faucher

\section{(2) OpenEdition Journals}

Édition électronique

URL : https://journals.openedition.org/baefe/884

DOI : $10.4000 /$ baefe.884

ISSN : 2732-687X

Éditeur

ResEFE

\section{Référence électronique}

Bérangère Redon, Sylvain Dhennin, Claire Somaglino, Matthieu Vanpeene, Lorenzo Medini, Louis Dautais, Séverine Marchi, Rim Saleh, Joachim Le Bomin, Julie Marchand, Charlène Bouchaud, MennatAllah El Dorry, Clémence Pagnoux, Robert Ryndziewicz, Tomasz Herbich, Maël Crépy, Alexandre Rabot, Paul François, Clementina Caputo, Mikaël Pesenti, Mahmoud Samir et Thomas Faucher, «Taposiris Magna et Plinthine (2019) » [notice archéologique], Bulletin archéologique des Écoles françaises à l'étranger [En ligne], Égypte, mis en ligne le 01 janvier 2020, consulté le 28 juillet 2021 URL : http://journals.openedition.org/baefe/884; DOI : https://doi.org/10.4000/baefe.884

Ce document a été généré automatiquement le 28 juillet 2021.

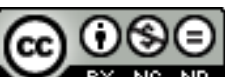

Le Bulletin archéologique des Écoles françaises à l'étranger est mise à disposition selon les termes de la Licence Creative Commons Attribution - Pas d'Utilisation Commerciale - Pas de Modification 4.0 International. 


\title{
Taposiris Magna et Plinthine (2019)
}

\author{
Bérangère Redon, Sylvain Dhennin, Claire Somaglino, Matthieu Vanpeene, \\ Lorenzo Medini, Louis Dautais, Séverine Marchi, Rim Saleh, Joachim \\ Le Bomin, Julie Marchand, Charlène Bouchaud, Mennat-Allah El Dorry, \\ Clémence Pagnoux, Robert Ryndziewicz, Tomasz Herbich, Maël Crépy, \\ Alexandre Rabot, Paul François, Clementina Caputo, Mikaël Pesenti, \\ Mahmoud Samir et Thomas Faucher
}

\section{NOTE DE L'AUTEUR}

Année de la campagne : 2019 (10 avril - 12 mai)

Numéro et intitulé de l'opération de terrain : 17111 - Taposiris Magna et Plinthine, deux villes de Maréotide

Composition de l'équipe de terrain : L'équipe était composée de Bérangère Redon (historienne, archéologue, CNRS, UMR 5189 HiSoMA), Marie-Françoise Boussac (historienne, université Paris Nanterre), Charlène Bouchaud (archéobotaniste, CNRSMNHN), Clementina Caputo (céramologue, Ruprecht-Karls-Universität Heidelberg), Maël Crépy (géographe et géomorphologue, CNRS, UMR 5189), Louis Dautais (doctorant en égyptologie, université Paul-Valéry Montpellier 3), Sylvain Dhennin (archéologue et égyptologue, CNRS, UMR 5189), Paul François (doctorant, architecte et ingénieur, université de Nantes), Joachim Le Bomin (archéologue, université Paris 1 PanthéonSorbonne), Julie Marchand (céramologue, CNRS, UMR 5189), Séverine Marchi (archéologue, CNRS, UMR 8167, équipe Mondes pharaoniques), Lorenzo Medini (archéologue et égyptologue, Ifao), Mikaël Pesenti (céramologue, université d'AixMarseille), Gaël Pollin (photographe, Ifao), Alexandre Rabot (spécialiste de SIG, UMR 5189 / université Lumière Lyon 2), Robert Ryndziewicz (géophysicien, PCMA), Rim Saleh (doctorante en archéologie, université Lumière Lyon 2), Claire Somaglino (archéologue et égyptologue, Sorbonne Université / UMR 8167) et Matthieu Vanpeene (doctorant, architecte, Ifao / EPHE-PSL). Nous étions accompagnés de 16 ouvriers venus de Gourna, d'une trentaine d'ouvriers locaux, de trois inspecteurs du ministère des Antiquités (MoA), Amany Shabaan, Hana Magdy Hassan Aly et Soha Abd el-Salam, et de 
Mohamed Khamis Ragab, inspecteur de la restauration. Ashraf Mohamed Abd elSamiah Abdel-Wahed a supervisé les prospections géophysiques et géomorphologiques. Ont participé à la mission, au titre du travail dans les magasins, au laboratoire d'étude des matériaux de l'Ifao ou en post-fouille : Charlène Bouchaud, Maël Crépy, MennatAllah El Dorry (archéobotaniste, Ifao / PCMA / MoA), Thomas Faucher (numismate, CNRS, Iramat-CEB), Tomasz Herbich (géophysicien, PCMA), Clémence Pagnoux (archéobotaniste, EfA), Mahmoud Samir (restaurateur indépendant), Gonca Cankardeşşenol (amphorologue, université d'Izmir). Les inspecteurs Amira Sabah et Mohamed Mohamed Mahmoud Ibrahim ont supervisé les travaux de Thomas Faucher et Mahmoud Samir à Shallalat.

Partenariats institutionnels : Comme chaque année, la mission était soutenue par le ministère de l'Europe et des Affaires étrangères (MEAE), ainsi que le CNRS (UMR 5189 HiSoMA, UMR 8167 Orient \& Méditerranée, équipe Mondes pharaoniques, MNHN). La mission a également reçu un important soutien de la fondation Arpamed pour ses travaux sur la vigne.

\section{Problématique et bilan des principaux résultats}

1 Situées à la marge/frontière occidentale de l'Égypte, les deux bourgades de Plinthine et Taposiris ont chacune connu des occupations longues et multiformes, Plinthine du Nouvel Empire à l'époque hellénistique, Taposiris prenant le relai jusqu'aux lendemains de la conquête arabe. Depuis 2012, la mission française de Taposiris Magna et Plinthine se concentre sur l'étude des vestiges d'époque ramesside à saïte de Plinthine, et du port et de la ville moyenne de Taposiris de l'époque romaine aux premières décennies de l'époque médiévale. Il s'agit de comprendre les mécanismes qui ont conduit au choix des deux sites pour leur implantation et aux raisons de leur dynamisme et périodes de déprise. L'étude des activités liées à la viticulture - encouragée par l'obtention d'une subvention spéciale du fonds Arpamed - entre pleinement dans ce cadre. La possibilité de produire du vin de qualité est en effet l'un des atouts de la région, qui a été exploité dès le Nouvel Empire à Plinthine.

2 La campagne de terrain 2019 s'est déroulée du 10 avril au 12 mai $^{1}$. Quatre secteurs ont été fouillés à Plinthine (secteur du temple, fouloir saïte, villa viticole ptolémaïque et habitat saïte) ainsi que les abords des thermes de Taposiris. Nous avons aussi travaillé sur le petit site satellite de Rahim, situé à $900 \mathrm{~m}$ de Plinthine, près du lac (fig. 1). Des opérations ponctuelles ont été conduites dans le port de Taposiris, pour dater les aménagements portuaires et la mise en œuvre du canal/port, en parallèle avec une prospection géomorphologique et des sondages à la tarière. À Plinthine et Taposiris, la prospection géomagnétique engagée en 2004-2005, puis en 2015, a été poursuivie, notamment dans la ville ptolémaïque de Plinthine (qui est dévorée par les figuiers) et aux abords des thermes. En parallèle avec la mission de terrain, les objets en métal mis au jour par la mission depuis une dizaine d'années ont été restaurés. À côté d'une dizaine d'objets divers (bols, bagues), 260 monnaies ont été nettoyées. Elles ont été étudiées en mai 2019. Enfin, une mission d'étude archéobotanique a été conduite sur le terrain en mai. Elle avait été précédée d'une mission d'identification des espèces conduite au Caire en janvier, mars et juillet 2019 et a été suivie d'une mission d'étude des variétés de raisin, en septembre 2019. En novembre enfin, a eu lieu une mission 
d'étude des prélèvements effectués à la tarière, pour caractériser les dépôts lacustres du port de Taposiris et le terroir viticole de Plinthine.

Fig. 1. Localisation de tous les sites archéologiques de la concession de fouille de la MFTMP (A. Rabot, fond de carte GoogleMap).

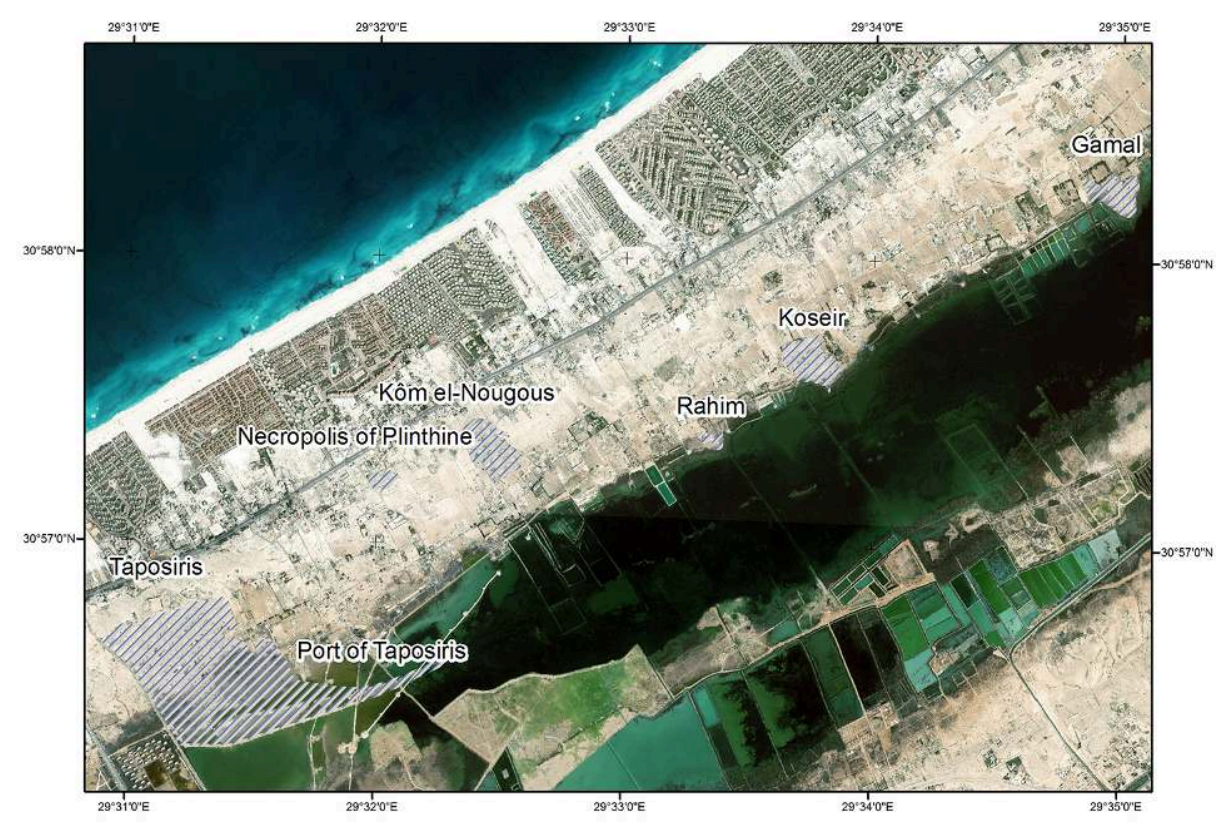

Sites of the Concession of the French Archaeological Mission of Taposiris, dir. B. Redon, 1:27 000, Red Belt

Sur le plan scientifique, les résultats de la campagne 2019 sont importants : le temple de Plinthine est désormais mieux compris, grâce à la découverte de dix inscriptions hiéroglyphiques, dont deux mentionnent le nom de Ramsès II. L'étude de la viticulture à Plinthine a également progressé, avec la multiplication des études archéobotaniques et la fouille du bâtiment au fouloir (secteur 6), remarquablement préservé et organisé. La villa viticole de Plinthine a été presque entièrement fouillée ; son abandon date de la fin de l'époque ptolémaïque. Enfin, 2019 a vu également l'achèvement de la fouille d'un quartier d'habitat saïte situé au sud du kôm, qui a connu de multiples phases de construction et de réaménagement, avant un abandon et le creusement d'une vaste fosse à l'époque ptolémaïque. L'étude de la céramique et des petits objets est en cours et illustre le dynamisme économique de la bourgade, notamment à l'époque saïte. À Taposiris, la fouille des abords des thermes a révélé la présence de plusieurs boutiques, organisées au sein d'un même édifice ou selon un même programme de construction. Le matériel associé a été entièrement étudié, dont un lot de céramiques provenant d'Abou Mina et sans doute emportées avec eux par des pèlerins. Enfin, l'abandon du site de Rahim a pu être daté du vile siècle apr.J.-C. tandis que son occupation pourrait remonter à l'époque ptolémaïque. Un édifice thermal a été dégagé ; il est bien préservé et devait être alimenté par une saqiah. Un entrepôt a également été identifié à proximité. Il est remarquablement construit et date de l'époque ptolémaïque. 
Enfin, pour restituer ces résultats au grand public, un travail de modélisation 3D du kôm de Plinthine a été engagé sur le terrain, avec l'acquisition de courbes de niveau, la prise de photogrammétrie, la centralisation des données (relevés architecturaux) récoltées depuis 2012. Un architecte/ingénieur travaille actuellement à la création et à la publication en ligne $d u$ modèle. On peut d'ores et déjà visiter, au moyen de lunettes 3D, le fouloir saïte de Plinthine en réalité virtuelle.

\section{Travaux de terrain à Plinthine et Taposiris}

\subsection{Fouilles à Plinthine}

(fig. 2)

\subsubsection{Secteur 7 : le temple}

Sylvain Dhennin, Claire Somaglino avec Matthieu Vanpeene et Lorenzo Medini

Fig. 2. Plan du kôm de Plinthine en fin de campagne 2019.

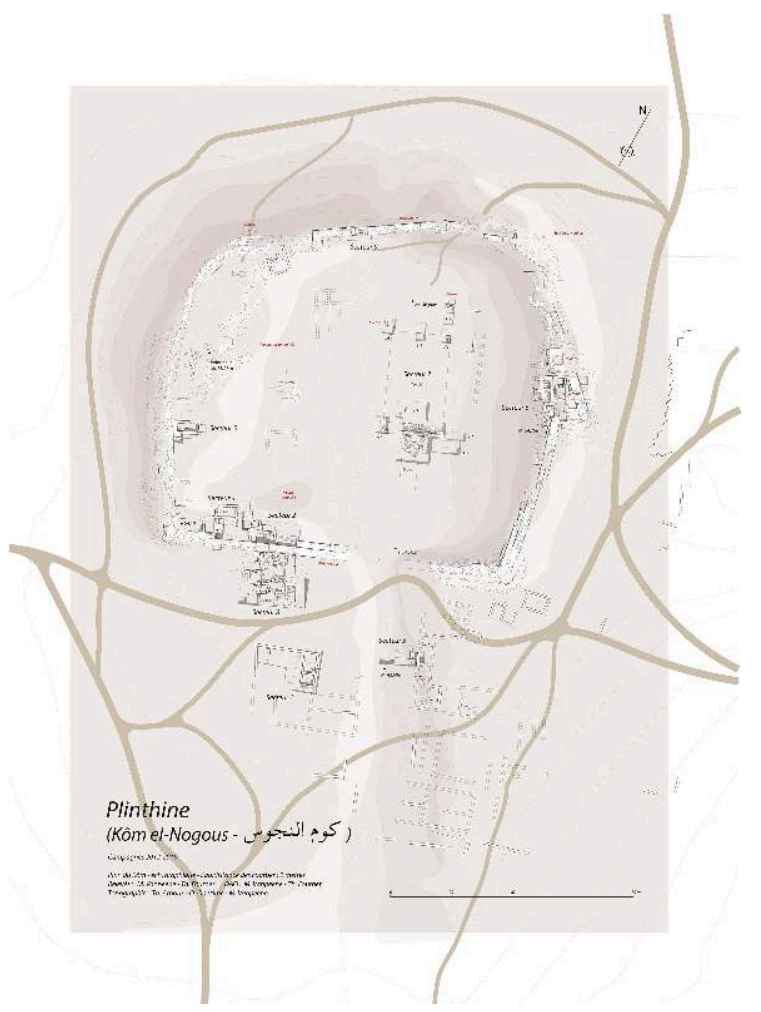

(C) MFTMP, DAO M. Vanpeene, Ifao. 17111_2019_NDMCN_002

La saison 2019 est la quatrième année de fouilles dans le secteur 7, situé dans la grande dépression qui occupe le centre du kôm. Dans cette zone, les fondations d'un grand bâtiment en pierre, qui doit être identifié à un temple, ainsi que des niveaux d'occupation du début de la Troisième Période intermédiaire ont été mis au jour au cours des trois précédentes saisons. L'objectif principal cette année était de localiser les angles du niveau de fondation du bâtiment, afin de déterminer ses dimensions et sa mise en œuvre, ainsi que de mieux comprendre le processus de destruction et de réutilisation des pierres de cet édifice. Ainsi, nous avons continué la fouille dans l'un 
des sondages-tests ouverts l'année dernière (sondage 3 ) et nous en avons ouvert trois autres (sondages 4,5 et 6 ).

Dans le sondage 3, situé au nord-est du secteur 7, l'angle nord-est du temple a été localisé ; il se matérialise par un creusement à angle droit dans le rocher, les blocs de l'édifice ayant ici été entièrement récupérés (fig. 3). Les fouilles de la zone ont également permis de mettre au jour des installations post-temple, qui témoignent des différentes étapes du travail effectué pour récupérer les blocs de ce monument. Pour contenir les blocs rejetés et les éclats de pierre, un mur orienté nord-sud a ainsi été construit en arrière de l'angle de ce qui était le temple (en raison de la pente, ces rejets auraient gêné les travaux qui avaient lieu au centre du kôm).

Fig. 3. Plinthine, sondage 3 à l'issue de la campagne 2018 (vue nord-sud).

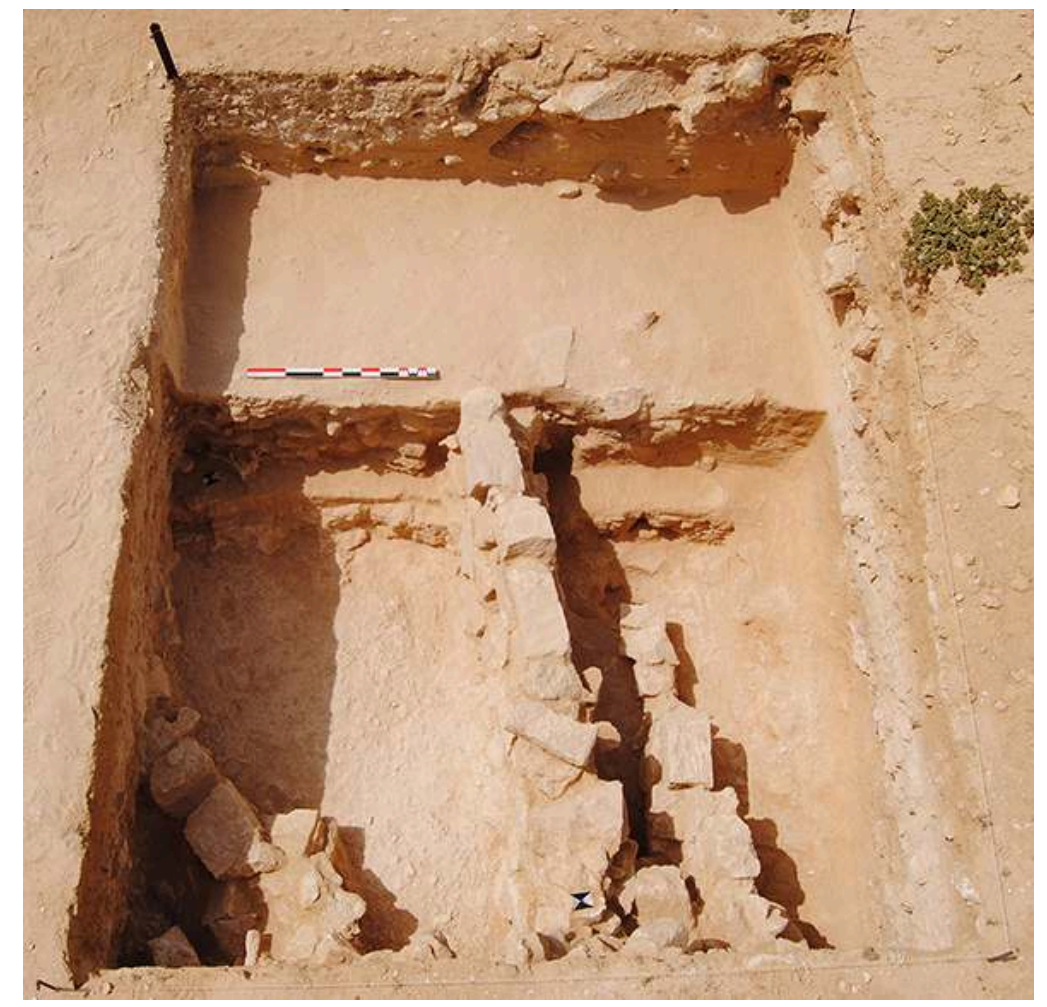

(c) MFTMP / L. Medini, Ifao. 17111_2019_NDMPF_001

7 Dans le sondage 4, situé à l'angle nord-ouest du kôm, une épaisse couche de destruction du temple, datée de l'époque romaine, a été mise au jour. Elle recouvrait une citerne de 2,50 $\mathrm{m}$ de profondeur, creusée dans la roche-mère et associée à un mur (fig. 4). Ce mur est construit avec des blocs réutilisés, dont un portant des décors. Nous avons dégagé l'angle nord-ouest du temple, également taillé dans le roc. Dans le remplissage de la citerne, plusieurs blocs décorés ainsi qu'une statue ont été mis au jour. Les sept blocs, en relief dans le creux, sont d'époque ramesside, plus précisément du règne de Ramsès II, selon les cartouches qui portent le nom de ce roi. Les blocs PO 664 et 667 donnent des parties du nom de naissance du roi ( $\left.R^{\prime}-m s-s w-m r y-J m n\right)$, alors que le bloc PO 675 donne une partie de son nom de trône (stp-n-R $R^{\prime}$. L'utilisation de la pierre locale permet de supposer qu'un temple a été construit à Plinthine ou à proximité pendant le règne de ce pharaon. Outre ces blocs, un buste de reine (?), en grès local, a été mis au 
jour (PO 690, fig. 5). La pierre est mal conservée, en raison de sa mauvaise qualité et du fait qu'elle est restée dans un environnement humide durant des centaines d'années.

Fig. 4. Plinthine, sondage 4 en fin de fouille, vue nord-sud.

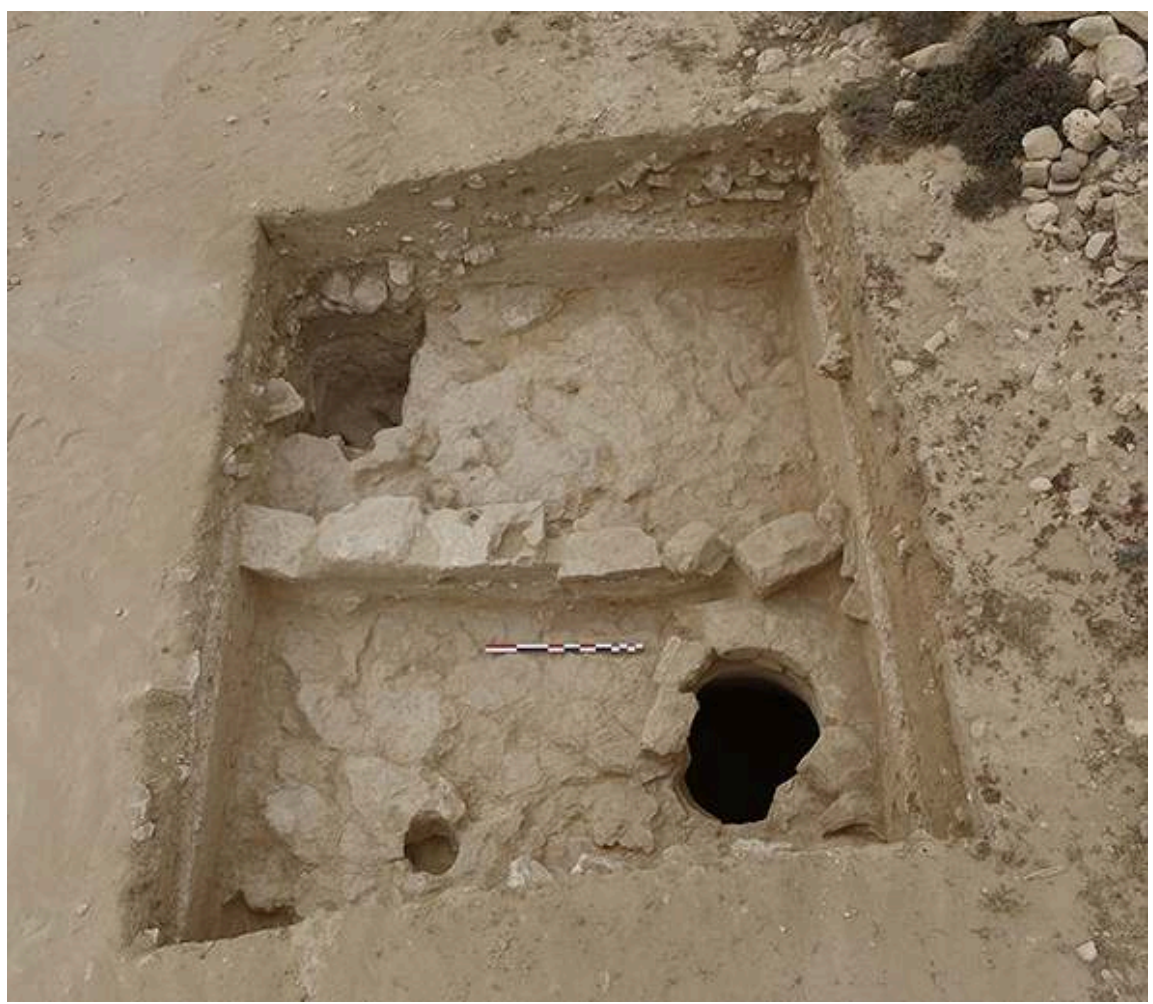

(c) MFTMP / Ifao, G. Pollin. 17111_2019_NDMPF_002 
Fig. 5. PO 690.

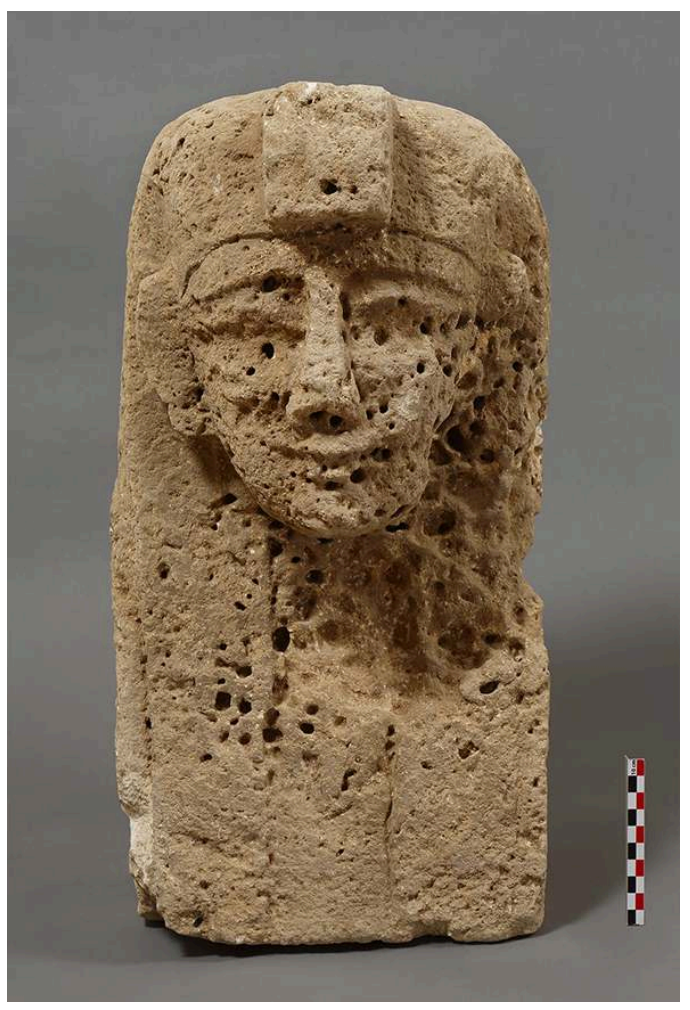

(c) MFTMP / Ifao, G. Pollin. 17111_2019_NDMPM_001

\subsubsection{Secteur 6 : le fouloir saïte}

Bérangère Redon, Louis Dautais avec Matthieu Vanpeene

Le secteur 6, fouillé depuis 2016, est situé au sommet de la partie orientale du kôm de Plinthine. On y a mis au jour un édifice abritant un fouloir daté du début de la $\mathrm{XXVI}^{\mathrm{e}}$ dynastie (voir les rapports précédents). Dans le prolongement de la campagne 2018, la saison 2019 a été consacrée à l'exploration de la partie sud du bâtiment. Nous voulions également savoir dans quel contexte urbain se développait cet édifice et si d'autres bâtiments lui étaient liés. Il s'agissait enfin de mieux cerner la chronologie des diverses occupations qui se sont succédé dans la zone après l'abandon du fouloir. 
Fig. 6. Plan du secteur 6 de Plinthine.

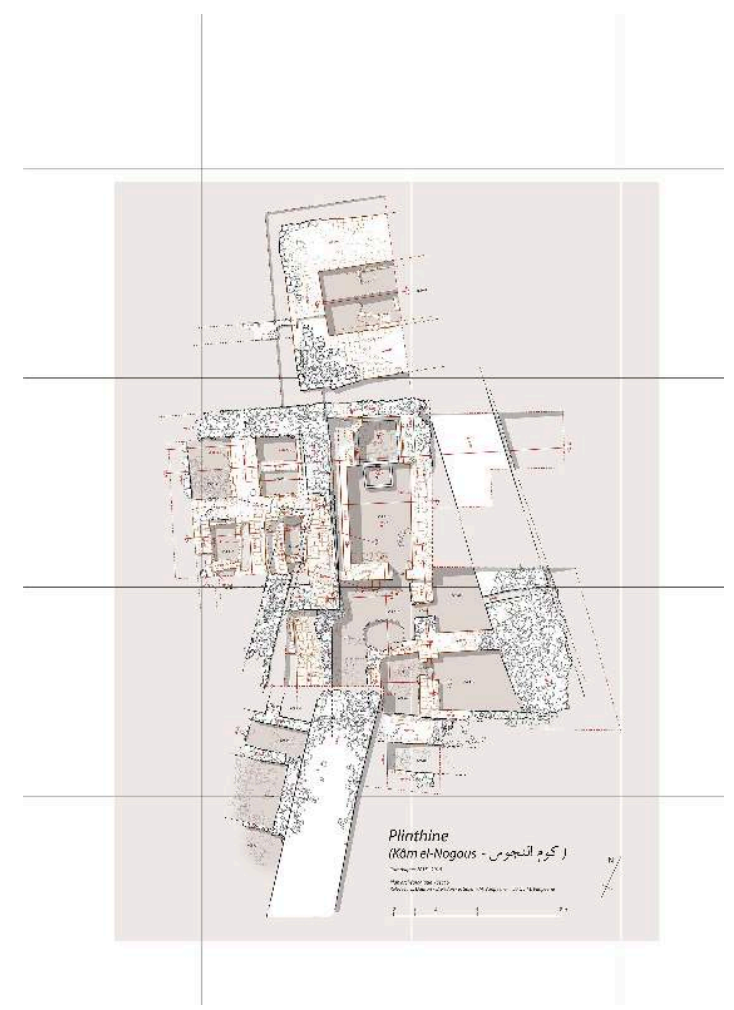

Fig. 7. Plinthine, vue générale du secteur 6 , vue vers le sud.

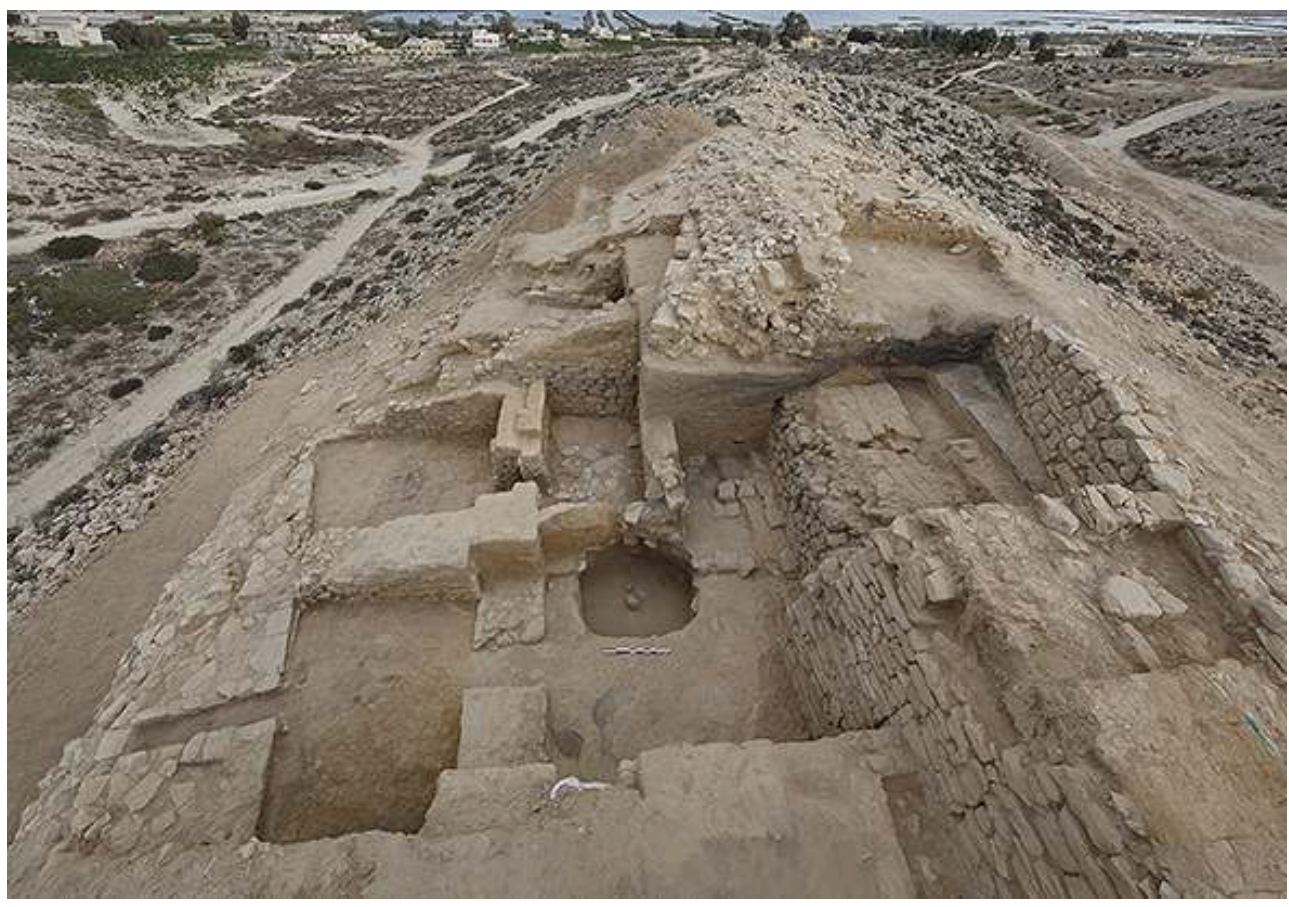

(C) MFTMP / Ifao, G. Pollin. 17111_2019_NDMPF_003

9 Au cours de cette saison, nous avons pu entièrement fouiller les pièces 617, 618 et 619 (fig. 6-7), qui font partie du grand bâtiment dans lequel prenait place le fouloir. La 
pièce 619 , située au sud-est du secteur, est probablement construite en même temps que le fouloir. Il ouvre à l'ouest par une porte bien faite, équipée d'un seuil et d'un emmarchement. Au moment de leur construction (au milieu du viI ${ }^{\mathrm{e}}$ siècle av. J.-C. ?), les deux bâtiments ouvraient sur un espace vide, peut-être une place ou une rue (espace 617). Par la suite, la zone est bornée à l'est par un grand mur (MR 619) qui sert de mur de soutènement. Il est très large (environ 3,10 m) et mesure plus de 15,50 $\mathrm{m}$ de long. Au cours de cette seconde phase de construction ou juste après, deux murs sont bâtis au sud et à l'ouest de la zone (MR 654 et MR 658); par conséquent, le fouloir (pièce 611) et la pièce 619 font désormais partie d'un seul et même bâtiment. Le hall 617 n'est plus une cour, mais le hall d'entrée du fouloir. Il ouvre au sud sur une petite pièce ou plutôt un espace (pièce 621) équipé d'une canalisation ménagée dans la maçonnerie du mur MR 619, pour évacuer les eaux usées et probablement pour permettre au fouloir d'être nettoyé à grande eau après les vendanges. Le fouloir est abandonné, ainsi que les pièces 617 et 621 , au vie siècle av. J.-C. La maison adjacente au fouloir (BAT 601) est alors toujours occupée et ses habitants prennent l'habitude de jeter leurs ordures (DEP 601) à l'extérieur de la maison, à l'est, au sommet de la démolition des pièces 617, 618, 619, 621 et du fouloir (pièce 611). Ce grand dépotoir a livré un matériel céramique et faunique important, vestiges de la vie quotidienne des habitants de la maison. On a aussi identifié de nombreux pépins de raisin dans la décharge, ce qui prouve que les activités viticoles se sont poursuivies même après l'abandon du fouloir. À la fin de la période qui voit la formation du dépotoir près de la maison BAT 601, plusieurs fours sont construits et reconstruits au sud de la maison (à l'abri de son mur sud qui protégeait la zone du vent). Ils sont très grossièrement construits (formés de quelques briques crues sans véritable organisation), mais leur activité est remarquable. Ils étaient probablement utilisés pour la cuisine. Au cours de cette phase, le grand mur MR 601 (2,73 m de large) est construit, probablement à la fin du $\mathrm{VI}^{\mathrm{e}}$ siècle av. J.-C. Enfin, plusieurs petites pièces sont construites contre le mur MR 601, au sud de la zone 6, ainsi que trois silos en briques crues. Quatre de ces pièces ont été fouillées cette année (pièces 603, 604, 605 et 606). Leurs sols étaient mal conservés et aucune couche d'occupation n'a été trouvée. Seul leur abandon peut être daté de la fin du $\mathrm{VI}^{\mathrm{e}}$ ou même du début du v siècle av. J.-C.

\subsubsection{Secteur 8 : un quartier saïte d'habitation}

Séverine Marchi, Rim Saleh 
Fig. 8. Plinthine, secteurs 2 sud et 8 , vue d'ensemble vers le sud-ouest.

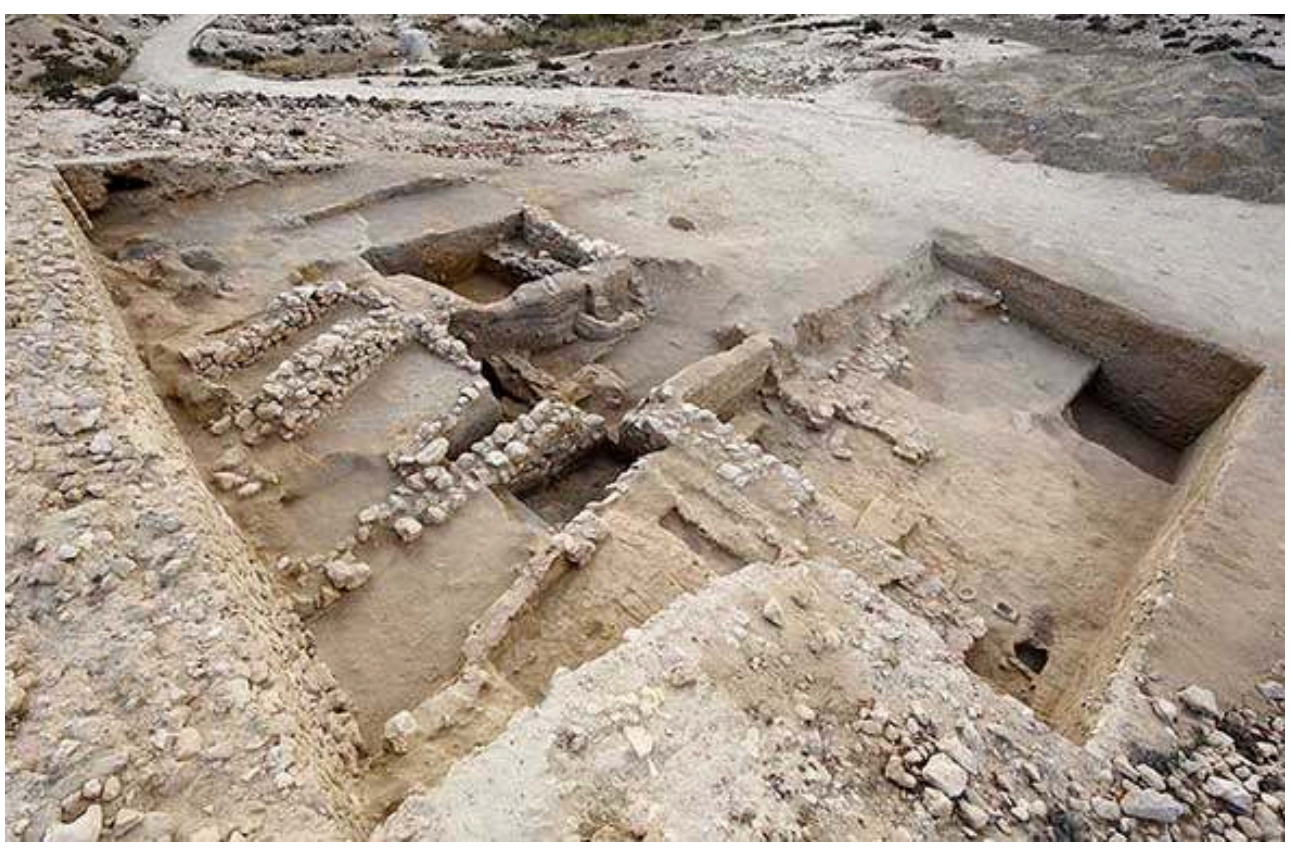

(c) MFTMP / Ifao, G. Pollin. 17111_2019_NDMPF_004

Fig. 9. Plinthine, secteur 8 . La faille dans la coupe C63.

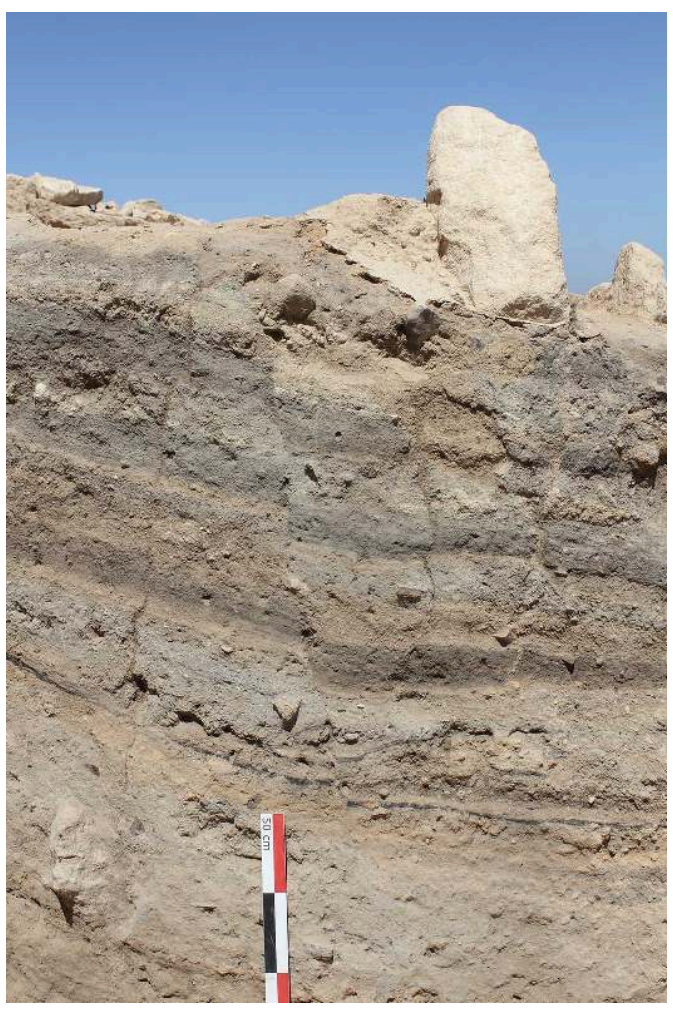

(c) MFTMP / S. Marchi. 17111_2019_NDMPF_005

10 Les opérations conduites entre 2013 et 2018 dans les secteurs 2 sud et 8 , au sud du mur d'enceinte ptolémaïque, avaient pour objectif de mieux comprendre la topographie de l'établissement dans la partie méridionale du kôm et d'établir la chronologie de son 
occupation. Le programme de fouille de cette année devait compléter les résultats jusqu'à présent obtenus en poursuivant le dégagement des vestiges mis au jour en 2018 et en essayant de corréler les niveaux et les phases d'occupation individualisés dans chaque secteur (fig. 8). Nous avons donc repris la fouille des niveaux situés à l'ouest, notamment ceux appartenant aux phases les plus anciennes, et tenté de relier stratigraphiquement les quatre phases d'occupation mises en évidence dans le secteur 8 aux six observées dans le secteur 2 sud. Une surface globale de $170 \mathrm{~m}^{2}$ a été rouverte. Le secteur 2 sud a été nettoyé pour compléter les plans de certains aménagements, pour vérifier les relations entre les vestiges et préciser les niveaux de construction des bâtiments. Ponctuellement, la fouille a été menée en profondeur afin d'atteindre les couches anciennes et aboutir à une meilleure compréhension du cadre chronologique grâce à l'analyse du mobilier céramique récolté. Plusieurs coupes stratigraphiques ont été réalisées pour favoriser la compréhension de phénomènes constructifs complexes induits par la topographie du site et correspondant à un habitat de pente. L'une des coupes a par ailleurs révélé l'existence d'un épisode sismique (fig. 9). Il se traduit par un net décrochement des dépôts archéologiques à l'emplacement d'une faille que l'on peut suivre sur près de $6 \mathrm{~m}$. Une analyse approfondie du mobilier céramique provenant des niveaux où la faille a été repérée permettra peut-être de préciser le moment où le site aurait été touché par un tremblement de terre qui a pu avoir des conséquences à plus grande échelle.

\subsubsection{Secteur 10 : la villa viticole ptolémaïque}

Louis Dautais, Rim Saleh

11 Dans le cadre du programme d'étude de la viticulture à Plinthine, nous avons repris la fouille d'une villa viticole construite autour d'un fouloir (secteur 10) et située au nord de la nécropole hellénistique de Plinthine. L'objectif principal de cette courte mission était d'établir un plan du bâtiment et d'en déterminer la disposition générale. D'une forme relativement carrée $(14,35 \times 14,46 \mathrm{~m})$, cet ensemble architectural est construit en blocs de grès, une pierre locale facilement accessible (une carrière se situe juste au sud). Les murs extérieurs de la villa sont plus larges que les murs internes. Seule la pièce accueillant le fouloir (1007) est construite en grand appareil constitué de gros blocs liés au mortier (fig. 10). Le bâtiment est directement construit sur le substrat rocheux, affleurant partout. Une ouverture percée dans le mur sud de la pièce 1003, non loin du niveau du sol, a été découverte; sa fonction n'est pas assurée (évacuation?). Les pièces étaient comblées de couches de sable et de grès consécutives à leur abandon (elles ont livré très peu de matériel, à l'exception de la pièce sudouest 1004). Une fine couche de sable jaune recouvrait le rocher dans toutes les pièces ; il devait servir de niveau de circulation dans les pièces de la villa. 
Fig. 10. Plinthine, secteur 10. Vue générale de l'établissement agricole depuis le nord-est.

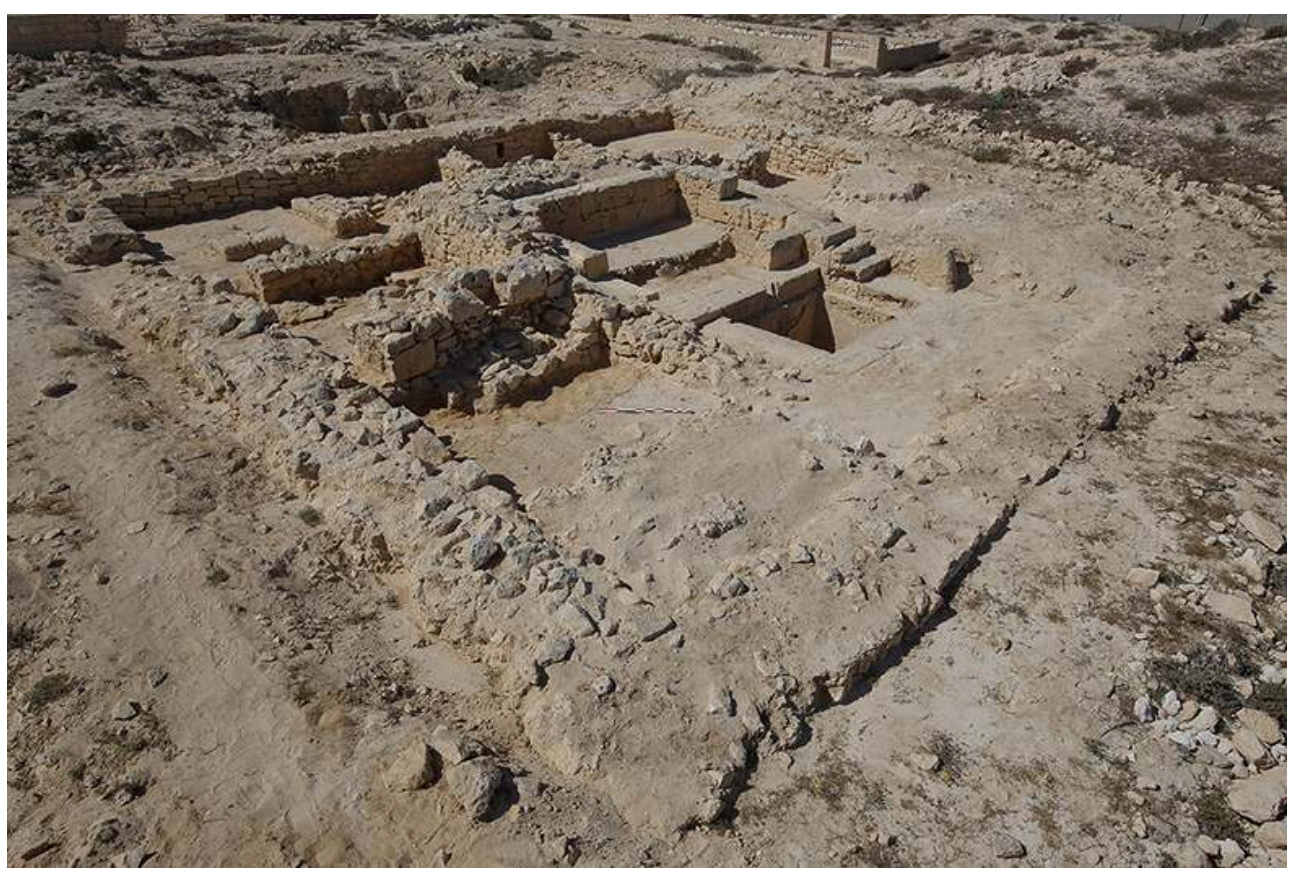

(c) MFTMP / Ifao, G. Pollin. 17111_2019_NDMPF_006

\subsection{Fouilles à Taposiris}

\subsubsection{Secteur 13 : les bains byzantins de la ville moyenne}

Joachim Le Bomin, Julie Marchand, Matthieu Vanpeene

Le but de la septième campagne de fouille des thermes de Taposiris était de finaliser le plan de la dernière phase du bâtiment (remanié au milieu du viI ${ }^{\mathrm{e}}$ siècle apr. J.-C.) et de commencer les fouilles des alentours des bains (fig. 11). 
Fig. 11. Taposiris, plan général du secteur 13 avec l'emplacement des sondages 2019.

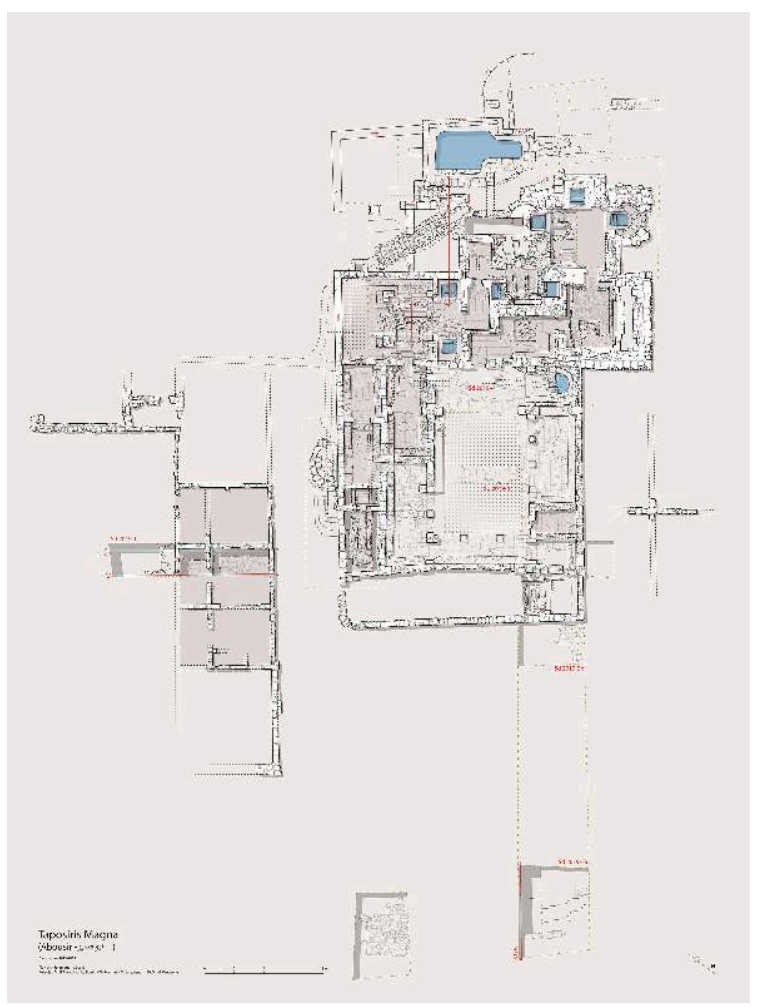

(c) MFTMP, DAO M. Vanpeene. 17111_2019_NDMCN_004

13 Dans les thermes, le puits PUI 002 situé dans la partie orientale de la cour COU 001 a été exploré. Dans la même cour, deux petits sondages ont été pratiqués pour dégager le sol au centre de la pièce et une canalisation dans la partie ouest.

Outre le nettoyage de la surface de la partie sud des bains, deux sondages ont été réalisés à l'extérieur des thermes :

- Le premier (sd 2019-1), de 10,50 ×3 m $(\mathrm{N} / \mathrm{S} \times \mathrm{E} / \mathrm{O})$, est localisé au sud des thermes, à l'emplacement de magasins datés du vi ${ }^{\mathrm{e}}$ siècle apr. J.-C.

- Le second (sd 2019-2) est un sondage est/ouest de 25,50 × 4,50 m, à l'est des thermes, dans la grande rue nord/sud qui conduisait du temple d'Osiris au port. Seules les extrémités ouest (vers l'esplanade des bains) et est (voie nord/sud) du sondage ont été explorées.

\subsubsection{Secteurs $16,18,19$ : sondages ciblés dans le port}

Joachim Le Bomin, Julie Marchand 
Fig. 12. Plan général de Taposiris avec la localisation des secteurs fouillés en 2019.

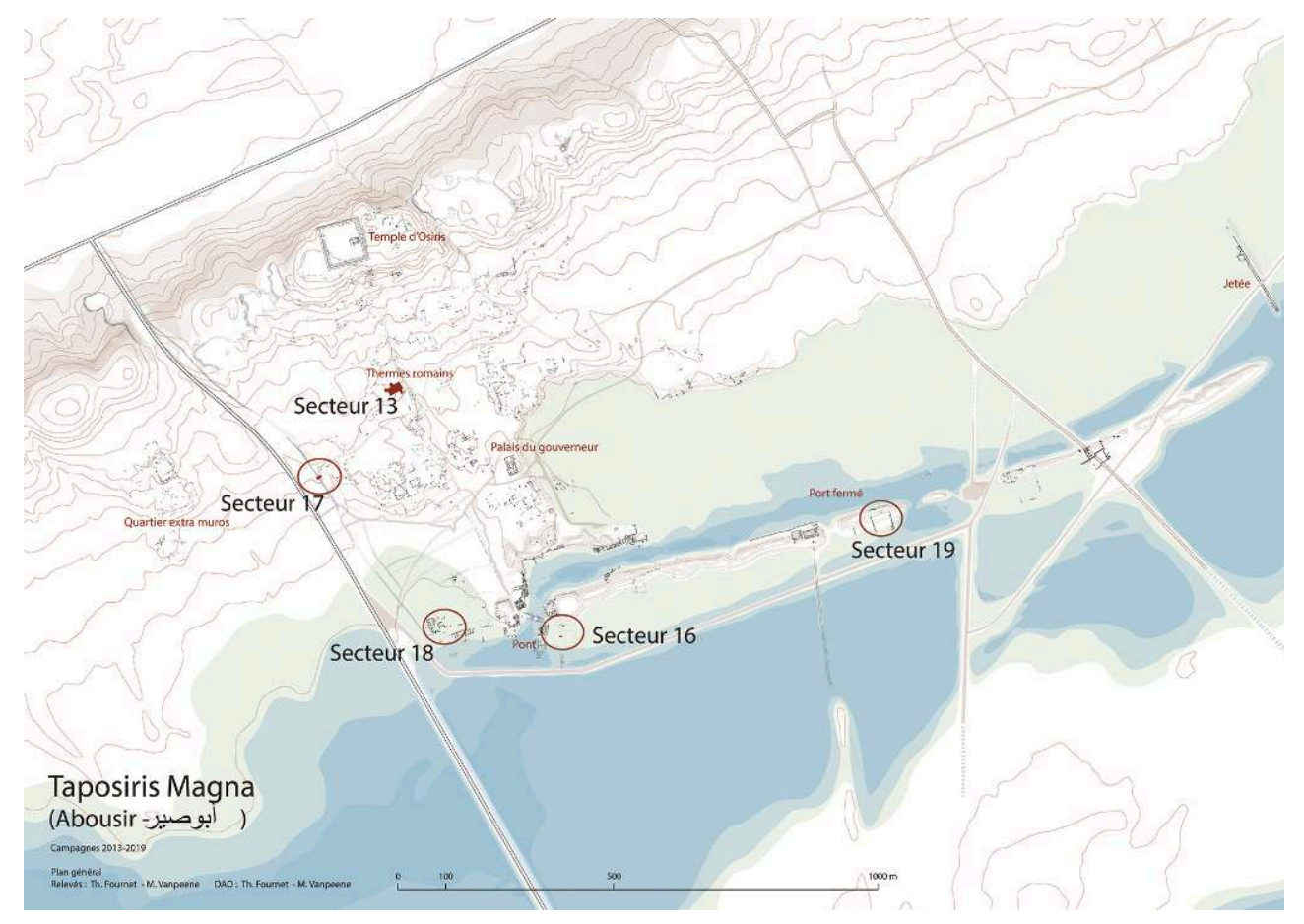

(c) MFTMP / DAO T. Fournet, M. Vanpeene. 17111_2019_NDMCN_005

Dans le cadre de l'étude du système portuaire de Taposiris, une série de sondages a été réalisée du 20 avril au 2 mai 2019 (fig. 12) :

- Secteur 16 : deux sondages $(6 \times 2 \mathrm{~m}$ et $2 \times 2 \mathrm{~m})$ ont été ouverts au sud-est du pont; ils avaient pour objectif de dégager un entrepôt repéré lors de prospections géomagnétiques. La nappe phréatique a été rapidement atteinte, nous empêchant de continuer les travaux.

- Secteur 18 : un sondage de $2 \times 2 \mathrm{~m}$ a été réalisé au cœur d'un bâtiment non identifié dans la partie sud-ouest de la ville, au bord du lac. Comme dans le secteur 16 , la nappe phréatique a toutefois été rapidement atteinte à environ $30 \mathrm{~cm}$ de profondeur sous la surface.

- Secteur 19 : dans la partie est de la jetée, au sud de celle-ci, un sondage a été effectué dans l'angle nord d'un entrepôt $(4,30 \times 2,40 \mathrm{~m})$; son abandon a été daté du III'e siècle apr. J.-C.

\subsection{Relevé et premières fouilles à Rahim}

Joachim Le Bomin, Julie Marchand, Matthieu Vanpeene

Rahim est un site situé à environ deux kilomètres à l'est de Kôm el-Nogous/Plinthine, au bord du lac Mariout. Il fait partie de la concession de la mission française de Taposiris Magna et Plinthine depuis 1998 et a fait l'objet de relevés topographiques en 2000 et 2001. En 2018, une couverture photographique et une prospection céramique avaient été réalisées par la mission française. Ces travaux avaient montré que le site était probablement occupé depuis la période hellénistique jusqu'à la seconde moitié du $\mathrm{VII}^{\mathrm{e}}$ siècle. En 2019, des nettoyages ont été conduits, dans le but de dater et comprendre la fonction de deux grands édifices localisés au sud du site. Deux secteurs (secteur 1 au sud et secteur 2 au nord-ouest) correspondant aux deux kôms principaux ont été étudiés. Le nettoyage du secteur 1 a révélé la présence d'un établissement thermal (fig. 13) dont l'abandon date du milieu du vII ${ }^{e}$ siècle. Le nettoyage du secteur 2, à l'ouest 
du kôm nord, a révélé la présence d'espaces de stockage datés de la période hellénistique.

Fig. 13. Thermes de Rahim depuis le nord en fin d'intervention.

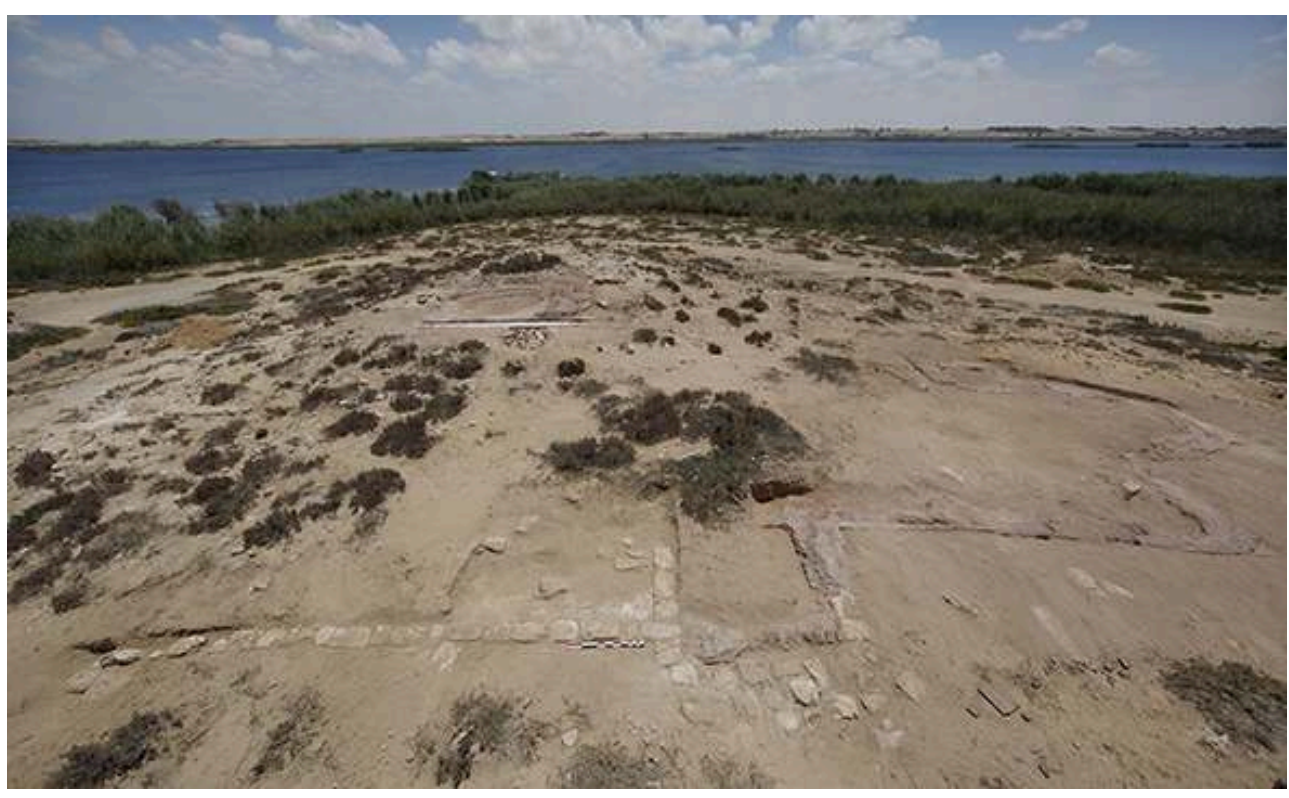

(c) MFTMP / Ifao, G. Pollin. 17111_2019_NDMPF_007

\section{3. Études environnementales}

\section{2. Étude des restes archéobotaniques de Taposiris et Plinthine}

3.2.1. Mission de terrain

Charlène Bouchaud 
Fig. 14. Machine à flottation.

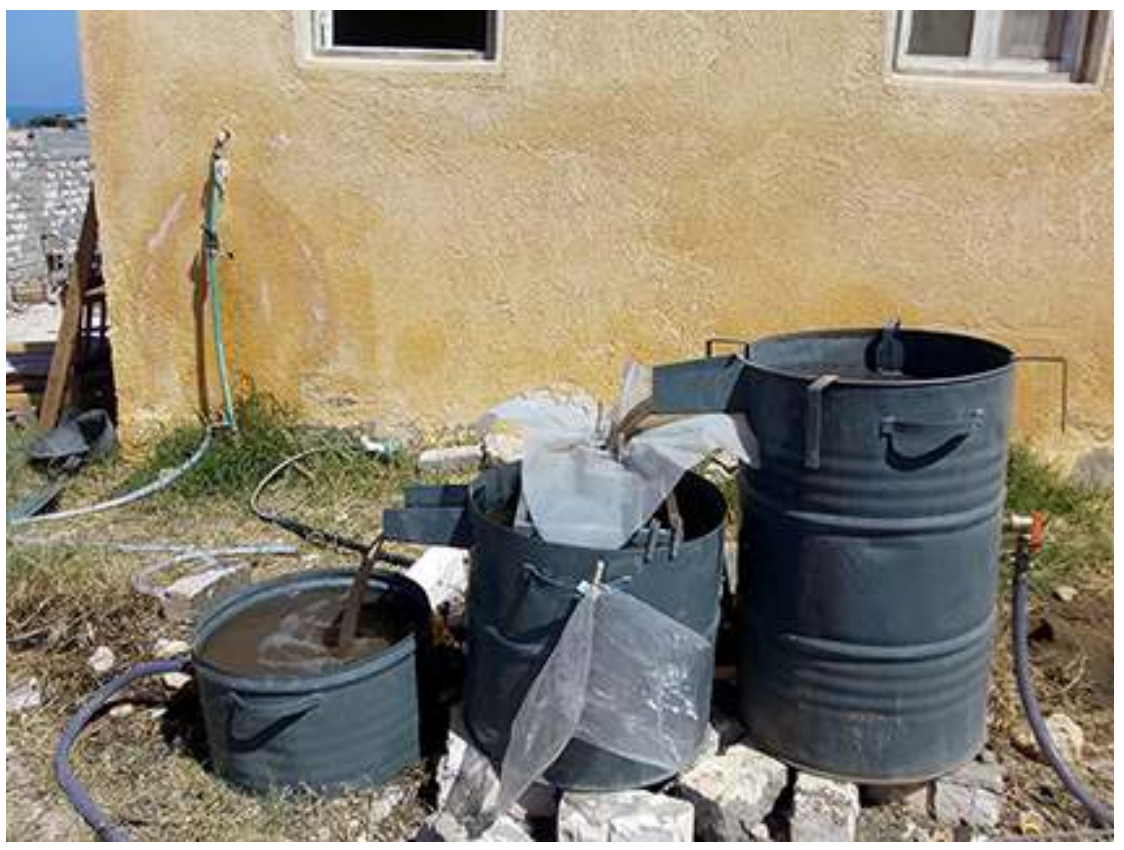

(C) MFTMP, C. Bouchaud. 17111_2019_NDMSE_001

17 Cette année, l'utilisation d'une machine à flottation, prêtée par l'Ifao, a grandement facilité le processus de tamisage (fig. 14), car elle permet de traiter plus rapidement une plus grande quantité d'échantillons. Cette machine est composée de trois fûts remplis d'eau alimentés par une pompe électrique hydraulique $(40 \mathrm{l} / \mathrm{min})$. Les prélèvements réalisés sur les sites sont lentement versés dans le premier fût. Le matériel carbonisé flotte à la surface et se déverse dans un second tamis à mailles de 0,3/0,5 $\mathrm{mm}$ placé juste au-dessus du second fût. L'eau passe ensuite par les deuxième et troisième fûts. Un filtre, placé sur l'évacuation du troisième fût, permet de filtrer l'eau qui est ensuite renvoyée, par la pompe, vers le premier fût. À l'intérieur, un tamis de 1 à $2 \mathrm{~mm}$ recueille le matériel archéologique (de la céramique et des os), qui sont triés à l'œil nu.

$\mathrm{Au}$ total, 54 échantillons provenant des différentes zones de fouilles (Plinthine: secteurs 6, 7, 8, 10; Taposiris Magna : secteurs 13, 19; Rahim) ont été traités en une semaine. Certaines identifications d'espèces ont été directement effectuées sur place, notamment les grains d'orge et de blé, les pépins de raisin, les lentilles. Néanmoins, toute l'identification n'a pas pu être réalisée en raison de l'absence d'outils spécifiques. Pour effectuer l'analyse, les macrorestes de plantes ont été exportés vers le laboratoire de l'Ifao à la fin de la campagne.

\subsection{2. Études en laboratoire}

Charlène Bouchaud, Mennat-Allah El Dorry, Clémence Pagnoux

Trois missions d'étude ont été réalisées en 2019 au laboratoire d'étude des matériaux de l'Ifao, grâce au soutien du fonds Arpamed. En plus de ces recherches, Mennat-Allah El Dorry a travaillé quatre semaines en juin et juillet sur le matériel de Plinthine, pour en continuer l'analyse et former trois laborantins du National Museum of Egyptian Civilisation du Caire. Ces travaux contribuent à préparer l'ouverture du laboratoire d'analyse de matériaux du musée en aidant à la formation de ses futurs chercheurs. 


\subsubsection{1. Étude de la vigne}

La présence de la vigne à Plinthine est désormais un fait établi. Les premiers résultats obtenus en 2018 ont montré le potentiel d'étude exceptionnel que représente l'analyse des résidus de raisin recueillis dans les sédiments de fouille. Les premières analyses s'étaient concentrées sur les secteurs des pressoirs d'époque saïte et ptolémaïque. L'étude de la forme des pépins archéologiques et leur comparaison avec des pépins actuels appartenant à de nombreuses variétés issues de différentes régions viticoles ont mis en évidence une grande diversité de morphotypes à Plinthine, tant à l'époque saïte qu'à l'époque ptolémaïque, et en particulier la présence de formes proches de la vigne sauvage.

Les résultats extrêmement prometteurs de cette première étude, ainsi que la découverte de nombreux pépins de raisin dans les nouveaux échantillons extraits des fouilles nous ont conduits à poursuivre l'étude morphométrique des pépins afin de préciser nos connaissances sur la diversité variétale de la vigne cultivée et son évolution à Plinthine au cours du $\mathrm{I}^{\mathrm{er}}$ millénaire av.J.-C. L'un des objectifs était d'analyser des échantillons datés des phases les moins représentées dans l'étude conduite en 2018, en particulier l'époque ptolémaïque. C'est pour cette raison qu'un ensemble de pépins provenant d'une couche ptolémaïque de Taposiris Magna a été étudié. En complément, nous avons également étudié cinq échantillons de Plinthine datés de la deuxième moitié $d u$ vil ${ }^{e}$ siècle / première moitié $d u v^{e}$ siècle av. J.-C. d'une part et $d u v^{e}$ siècle av. J.-C. d'autre part. Au total, 686 pépins provenant de six échantillons ont été photographiés. Cette nouvelle série de photographies a permis de compléter un lot particulièrement riche provenant de l'unité stratigraphique 6314 (DEP 601 = dépotoir présent dans la zone de fouloir saïte où plus de 600 pépins entiers ont été trouvés et photographiés). C'est la première fois qu'un ensemble archéologique numériquement aussi important provenant d'un seul contexte peut être étudié. Cette nouvelle perspective d'analyse permettra d'explorer le lien entre le nombre de pépins étudiés et le nombre de morphotypes observés, et de rechercher le nombre d'individus nécessaire à une description optimale de la diversité au sein d'un ensemble.

\subsubsection{Autour du raisin : l'agrobiodiversité de Plinthine et de Taposiris Magna}

Le raisin n'est pas la seule culture avérée à Plinthine. La découverte de vestiges de graines, fruits et bois brûlés, principalement dans les niveaux de la première moitié du $\mathrm{I}^{\mathrm{er}}$ millénaire av. J.-C., indique une agriculture variée, permettant de satisfaire au moins une partie des besoins alimentaires des populations humaines et non humaines vivant sur le site. Nous avions déjà mis en évidence en 2018 la présence d'orge, de blé amidonnier, de blé nu, de lentille, de pois, de pois chiche. Les quatorze nouveaux échantillons de graines et de fruits, ainsi que les sept échantillons de charbon de bois étudiés permettent d'allonger la liste de nouvelles plantes, incluant la figue, la vesce et un grand nombre de plantes sauvages poussant dans les champs, le long des chemins et autres espaces anthropisés, en milieu humide et désertique. Ces résultats soulignent la diversité des terrains exploités autour du site.

\subsection{Prospections géophysiques à Plinthine et Taposiris}

Robert Ryndziewicz avec Tomasz Herbich 
Les prospections géophysiques effectuées à Plinthine en 2019 prennent la suite de prospections réalisées en 2015 (2,2 ha). Cette année, une superficie totale d'environ 2,3 ha a été couverte (fig. 15).

Fig. 15. Plinthine, localisation des zones A, B et C prospectées en 2019 par R. Ryndziewicz.

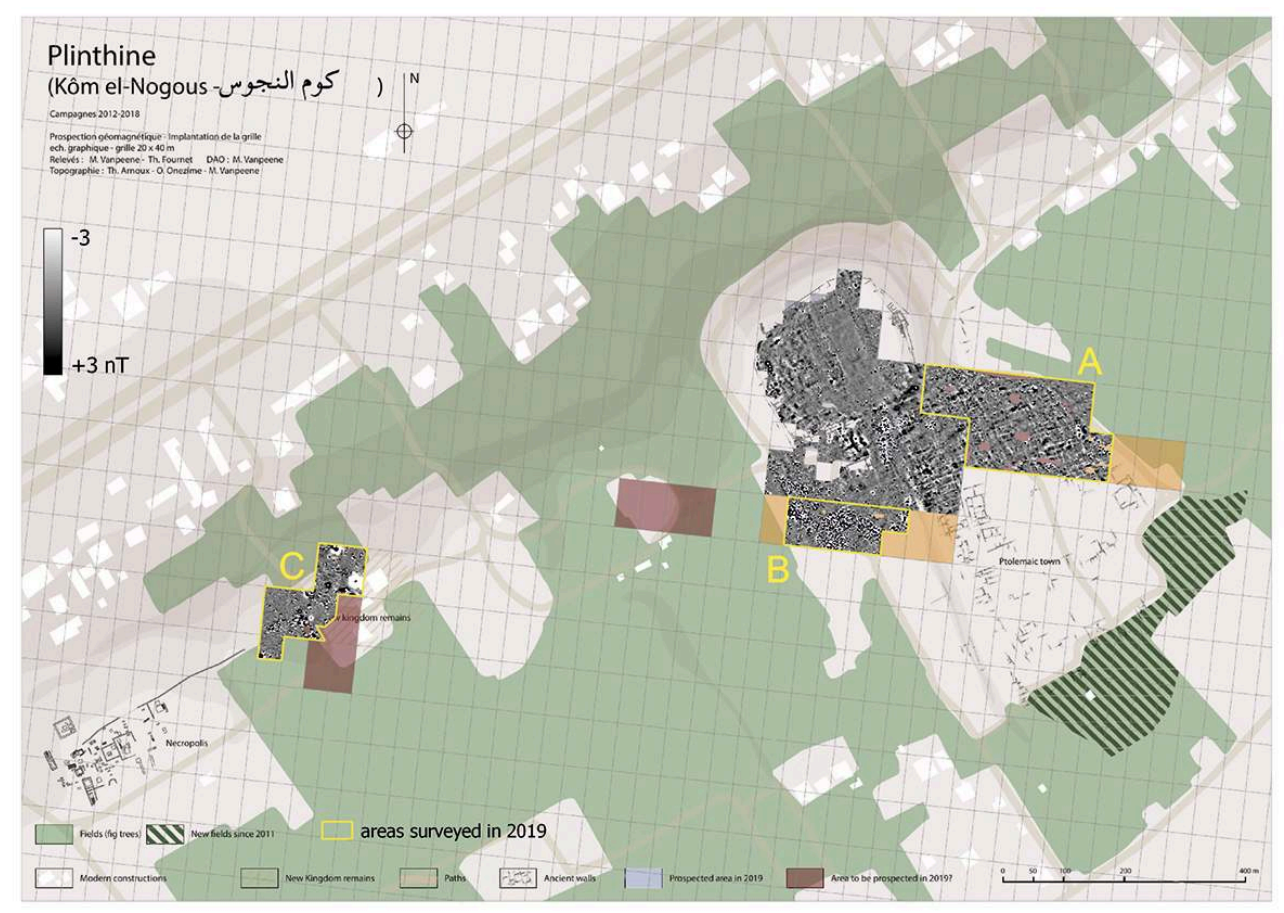

(C) MFTMP, M. Vanpeene, R. Ryndziewicz. 17111_2019_NDMCN_006

\begin{tabular}{|l|l|}
\hline Site & $\begin{array}{l}\text { Ptolemaic Town Plinthine, Kom el-Nogous, Taposiris Magna, } \\
\text { Egypt }\end{array}$ \\
\hline Prospection method & Magnetic (fluxgate gradiometer survey) \\
\hline Date of fieldwork & 24 April - 04 May, 2019 \\
\hline Instrument & Geoscan Research FM256 \\
\hline Sampling grid & $0,50 \times 0,25 \mathrm{~m}$ \\
\hline Resolution & $0,1 \mathrm{nT}$ \\
\hline Processing software & Geoscan Gesearch Geoplot 3.0 \\
\hline Field measurments and data \\
processing
\end{tabular}

Quatre zones ont été prospectées :

-zone nord-est de la ville ptolémaïque de Plinthine : la carte magnétique produite permet de reconstruire assez bien l'orientation générale des bâtiments et les plans de quelques bâtiments particuliers. Les murs construits en calcaire (non magnétique) sont visibles sous forme d'anomalies négatives dans un environnement plus magnétique ; 
- zone sud-ouest de la ville ptolémaïque de Plinthine : la carte magnétique n'a pas fourni de données sur les structures archéologiques en raison de la présence d'une grande quantité de déchets très magnétiques à la surface ;

- zone située entre le kôm et la nécropole de Plinthine : certaines anomalies peuvent être interprétées comme étant produites par des fours et probablement des petites structures régulières ;

- environs immédiats des bains byzantins de Taposiris Magna : la carte magnétique est très nette et permet de constater que de nombreux murs de la zone suivent la même orientation que les thermes du secteur 13. Certaines anomalies positives peuvent être interprétées comme des dépôts de cendres.

\subsection{Prospections géomorphologiques et analyses sédimentologiques}

Maël Crépy

\subsubsection{Mission de terrain}

Cette année, la mission avait deux objectifs principaux :

- étudier les différents «terroirs» de production viticole à Plinthine et à Rahim et leur contexte sédimentologique ;

- recueillir plus de données sur le niveau d'eau du lac Mariout à l'époque antique et ses fluctuations, en comparant les données archéologiques et historiques et les archives sédimentaires.

Prospections et coupes de sols nous ont permis d'identifier deux zones qui étaient probablement très avantageuses pour la culture du vin à Rahim et entre la nécropole de Plinthine et le Kôm el-Nogous. Les carottes sédimentaires et les profils de sol des fouilles effectuées dans l'ancien port de Taposiris ont permis de documenter les différents niveaux du lac Mariout aux époques hellénistique, romaine et byzantine. Au cours des travaux de terrain, 63 échantillons, dont 15 sections appartenant à 4 carottes (pratiquées à la tarière manuelle), ont été prélevés pour analyse.

\subsubsection{Analyses en laboratoire}

En septembre 2019, une combinaison de trois types d'analyse a été effectuée sur les échantillons issus des sondages à la tarière, au laboratoire d'analyse des matériaux de l'Ifao: granulométrie par tamisage (27 échantillons), destruction du gypse (13 échantillons), de la matière organique (13 échantillons) et des carbonates de calcium (9 échantillons) par perte au feu et observation des sédiments à la loupe binoculaire (27, fraction par fraction soit un total de 231 sous-échantillons). Les résultats de cette année ont notamment permis d'identifier plusieurs zones de cultures antiques avérées (entre le kôm de Plinthine et la nécropole ptolémaïque) ou potentielles (Rahim) dans le secteur. 


\section{SIG, 3D et modélisation}

\subsection{SIG}

Alexandre Rabot

La mission avait trois objectifs principaux :

- unifier le relevé topographique de l'ensemble du site (Taposiris et Plinthine) ;

- intégrer toutes les données dans un nouveau système général permettant de créer un SIG complet ;

- articuler les différents systèmes altimétriques des relevés précédents.

Pour atteindre ces objectifs, A. Rabot et M. Vanpeene ont enregistré les points de référence qui servent, depuis 1998, à localiser les travaux archéologiques, à la fois par GPS et par tachéomètre. Cette mise à plat nécessaire, après plus de vingt ans de fouille, permettra de disposer à moyen terme d'un SIG offrant une cohérence relative de tous les relevés archéologiques effectués jusqu’à présent, mais aussi de croiser les données de la mission avec d'autres, extérieures (comme les cartes du Survey of Egypt), et lorsqu'on travaille (comme M. Crépy, géomorphologue) à des niveaux très bas (proche du NMM) où le système altimétrique local apparaît inadapté (une dizaine de mètres audessus de l'altimétrie cartographique et du niveau moyen du lac Mariout actuel).

\subsection{Réalisation de photogrammétries et de modèles 3D à Plinthine}

Paul François

\subsubsection{Travaux de terrain}

La mission photogrammétrique s'est déroulée du 21 avril au 3 mai 2019 avec trois tâches principales : établir une reconstitution 3D multi-échelle du kôm de Plinthine ; produire des photographies photogrammétriques des secteurs archéologiques à la demande des archéologues; dessiner des blocs architecturaux et des objets au fur et à mesure de leur découverte.

31 La première tâche était l'objectif principal de la mission, pour comprendre la morphologie du site, ce qu'il était possible et nécessaire de réaliser sur le terrain compte tenu de nos objectifs. Il a été décidé qu'un nouveau relevé topographique représenterait un travail considérable pour produire peu de nouveaux résultats, voire aucun, d'où la décision de s'appuyer principalement sur des enquêtes précédentes et des campagnes photogrammétriques réalisées par A. Rabot et M. Vanpeene. La production du modèle 3D interactif multi-échelle et diachronique est en cours.

Les levés photogrammétriques et les dessins de blocs architecturaux ont été réalisés principalement sur les secteurs 6,7 et 10 , ce qui a permis de produire à la fois une orthophotographie des secteurs et des modèles 3D qui constitueront une ressource précieuse pour les futures reconstructions numériques. Un objet particulier a retenu notre attention: une statue de femme (reine?) mise au jour dans le secteur 7 pour laquelle nous avons produit un modèle tridimensionnel à haute résolution, adapté à la recherche, à la reconstruction et/ou à la vulgarisation (https:// taposiris.hypotheses.org/1171). 


\subsubsection{Restitution du fouloir saïte de Plinthine}

Le caractère exceptionnel de la découverte du fouloir saïte à Plinthine (secteur 6), de même que son bon état de conservation se prêtent particulièrement bien à une tentative de restitution, et ce d'autant plus que les environs immédiats du fouloir au sud ont fait l'objet d'un dégagement qui permet de mieux comprendre la relation de cet espace avec le tissu urbain local.

Les relevés photogrammétriques effectués lors des saisons 2018 (pour le fouloir proprement dit) et 2019 (pour les espaces au sud de la porte du fouloir) ont servi de base au travail de restitution, d'autant que les élévations des murs sont conservées sur plusieurs mètres, ce qui a grandement facilité le positionnement des éléments architecturaux (murs, portes, voûtes, etc.). Cette «extrusion » systématique du profil des murs a néanmoins rapidement montré ses limites au regard des problématiques de phasage de l'occupation du secteur, d'usage des installations du fouloir et de technique constructive. Pour aller plus loin, les photogrammétries ont été exportées sous la forme de modèles maillés et texturés depuis Agisoft Metashape. Le nombre de polygones a ensuite été décimé afin de permettre un affichage fluide suffisamment précis après import dans 3DS Max et lors de la visualisation en réalité virtuelle. L'ensemble de la modélisation volumique a été effectué sur 3DS Max, tandis que le rendu des textures et lumières a été réalisé grâce au moteur de rendu VRay, qui offre des outils d'éclairage physiquement réalistes. En raison de l'évolution rapide du modèle au gré des hypothèses archéologiques, nous avons fait le choix de ne faire appel qu'à des textures paramétriques (entièrement générées par ordinateur, après programmation): la création de ces textures a été fastidieuse, mais a permis un gain de temps appréciable lors des différentes modifications du modèle.

Afin de permettre aux archéologues de visualiser les hypothèses de restitution et de les discuter, nous avons mis en place une application de visite en réalité virtuelle à l'échelle 1 de la restitution des environs du fouloir, mais également du modèle photogrammétrique : le passage par simple pression sur une gâchette de la restitution à la réalité (ou virtualité) du terrain permet alors des échanges rapides et donc un modèle in fine de meilleure qualité. Cette expérimentation a été réalisée grâce au moteur Unity dans lequel l'ensemble des ressources 3D ont été incorporées, et à un masque de réalité virtuelle HTC Vive.

La restitution proposée ici (fig. 16) été réalisée après de nombreux échanges avec les archéologues, concernant la couverture de la pièce, sa restitution architecturale, ainsi que celle du mobilier céramique. Le modèle est accessible en ligne sur Sketchfab et la méthodologie ainsi que les choix opérés feront l'objet d'un prochain article. 
Fig. 16. Proposition de reconstitution du fouloir saïte de Plinthine.

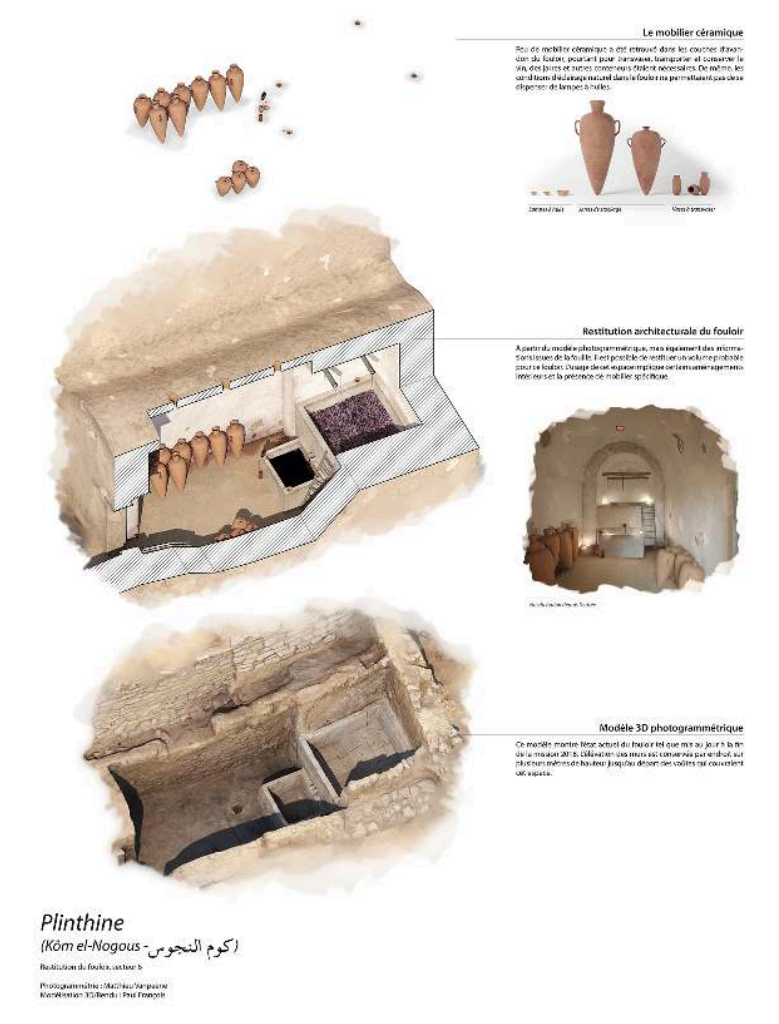

(c) MFTMP, P. François. 17111_2019_NDMPM_002

\section{5. Études de matériel}

Le matériel céramique découvert durant la mission de terrain a été étudié pendant la campagne de fouille par J. Marchand (Taposiris) et C. Caputo (Plinthine). Tous les petits objets et blocs architecturaux trouvés en 2019 à Taposiris et Plinthine ont été enregistrés et dessinés sur le terrain par J. Marchand, S. Marchi et P. François, avec l'aide de Rim Saleh pour l'enregistrement. Ils ont été photographiés par G. Pollin. Enfin, la majorité des objets métalliques entreposés par la mission au magasin de Shallalat ont été restaurés par Mahmoud Samir, ce qui a permis à T. Faucher de poursuivre l'étude des monnaies.

\section{1. Étude de la céramique de Plinthine}

Clementina Caputo avec Mikaël Pesenti

La céramique découverte au cours de la saison 2019 dans les secteurs 6, 7 et 8 n'a pu être que partiellement traitée, en raison de la grande quantité de matériel et de l'absence, pour des raisons personnelles, de M. Pesenti. Néanmoins, des types sélectionnés dans chaque secteur ont été dessinés et photographiés, ce qui permet de proposer ici un diagnostic chronologique et typologique. L'étude du matériel restant sera poursuivie l'année prochaine. 


\subsubsection{Secteur 6}

D'après une analyse préliminaire, la céramique trouvée dans le secteur 6 confirme une occupation conséquente à la période saïte. Le matériel trouvé dans les niveaux de surface et dans les niveaux correspondant à la dernière phase d'occupation (PCE 604-606 et fours) semble homogène et date principalement du milieu du vi ${ }^{\mathrm{e}}$ siècle jusqu'au premier quart du $v^{e}$ siècle av. J.-C. Il s'agit principalement de céramique produite localement, telle que des bols, des bassins, des cruches, des marmites, des mortiers et des dokkas. En outre, on note la présence dans les assemblages d'une forte proportion d'importations (amphores de Grèce et du Levant et céramique fine, notamment grecque).

40 Les fouilles ont également porté sur un vaste dépotoir (DEP 601), déjà fouillé en 2018. L'importance numérique de ce lot de céramique, mis au jour au cours des derniers jours de fouille, a empêché qu'il soit entièrement étudié. Son analyse préliminaire montre que la grande majorité des individus datent de la fin de la période saïte. Il convient de noter la présence dans certaines US situées à la base du dépotoir de quelques vases complets (deux pots de cuisson et deux petits récipients ovoïdes) qui pourraient remonter vers la fin du viI siècle av. J.-C.

\subsubsection{Secteur 7}

41 La céramique du secteur 7 semble appartenir à différents horizons chronologiques. Bien que certaines unités stratigraphiques semblent perturbées, en raison de la présence de types saïtes mélangés à des types romains, la majeure partie de la céramique est attribuable aux périodes romaine $\left(\mathrm{I}^{\mathrm{er}}-\mathrm{II}^{\mathrm{e}}\right.$ siècle) et romaine tardive ( $\mathrm{v}^{\mathrm{e}}$ siècle). La période romaine est bien représentée par des amphores égyptiennes de type 4 et 3 produites localement, de la vaisselle de table et de cuisine, ainsi que par des importations en provenance de Grèce orientale. Parmi les types datant de la période tardive romaine, il convient de noter la présence d'amphores protobyzantines LRA 1 de Chypre. L'un des niveaux les plus anciens documentés cette année contenait le premier moule à pain tubulaire attesté jusqu'à présent à Plinthine, daté du Nouvel Empire (XVIII dynastie). Il a été trouvé avec un tesson d'un petit vase décoré d'un motif typique de la poterie mycénienne (1350-1300 av. J.-C.).

\subsubsection{Secteur 8}

La plupart du matériel céramique trouvé cette année dans les US du secteur 8, malgré quelques rares intrusions, reste cohérent avec la date assignée pendant la saison 2018 à l'occupation principale de la zone : entre la fin de la Troisième Période intermédiaire et la période saïte. Il se compose principalement de vases de production locale, de table, de cuisine et de stockage. Là encore, comme pour le secteur 6 , il convient de noter la présence importante d'importations, notamment des amphores grecques (Lesbos, Chios, Clazomènes de Milet et Samos) et des jarres levantines (torpedo), ainsi que de la céramique fine grecque. Des amphores à anse de panier et des mortiers chypriotes complètent l'aperçu rapide du matériel importé. De la céramique ptolémaïque a été mise au jour dans le secteur 8 , notamment dans les niveaux de destruction de la zone. 


\subsubsection{Secteur 10} sélectionnés pour chaque unité stratigraphique ont été dessinés. Le matériel est peu abondant (environ 35 individus) et date de la période ptolémaïque ( $\left(\mathrm{III}^{\mathrm{e}} \mathrm{I}^{\mathrm{er}}\right.$ siècle av. J.C.). Les types les plus récurrents sont les amphores égyptiennes de type 2 , les bols et les assiettes convexes avec bord intérieur en pâte calcaire et alluviale (Egyptian Black Ware) et les marmites (chytrai et caccabé).

\subsection{Céramique de Taposiris}

Julie Marchand

\subsubsection{Secteur 16 : le port}

Les US de surface ont permis de recueillir quelques vestiges de l'occupation la plus tardive de la ville de Taposiris : ce sont ainsi des amphores LRA 5/6 de facture locale ainsi que des amphores tardives LRA 1 de Cilicie et de Chypre. Les niveaux inférieurs présentent une importante variété d'amphores égyptiennes, de type AE 3 du Delta et du Mariout et une AE 4 du Mariout, ainsi que des importations : une probable cilicienne ou un prototype assimilé, une Tripolitaine III et une amphore égéenne.

\subsubsection{Secteur 19 : Les entrepôts est}

La prospection de surface menée sur tout le secteur des entrepôts a permis de collecter plusieurs conteneurs, majoritairement des amphores. Les témoins égyptiens sont romains, de type $\mathrm{AE} 3$ en pâte calcaire et alluviale. Les importations sont assez nombreuses: LRA 3 de type B2, ainsi qu'une "pinched-handle». Un tesson de plat sigillé ESA a aussi été observé, ainsi que quelques plats de céramique commune en argile locale. Ils témoignent d'une occupation du secteur entre le III $^{\mathrm{e}}$ et le $\mathrm{v}^{\mathrm{e}}$ siècle. Dans le sondage ouvert dans deux salles de l'entrepôt, la céramique de la phase d'abandon date du tournant $d u v^{e}$ siècle. Une seule phase d'occupation a été fouillée dans la dernière des salles de l'entrepôt du sondage 19. Le matériel récolté se compose de trois amphores importées, ainsi qu'une amphore non identifiée pour le moment et une amphore de table. Cet assemblage serait à dater de la fin $\mathrm{du}_{\mathrm{Iv}}^{\mathrm{e}}$ siècle. Tout le matériel récolté est associé au stockage ou au reconditionnement du vin.

\subsubsection{Secteur 2 : entrepôts est byzantins (fouille 2018)}

L'unité stratigraphique 2000 (niveau d'abandon) des entrepôts byzantins situés à l'ouest des précédents, au débouché d'une ouverture/canal de direction nord-sud, sur la jetée de Taposiris (secteur 2), a été fouillée en 2018: cette US comporte un matériel extrêmement homogène datable entre le milieu du $\mathrm{VII}^{\mathrm{e}}$ et le début du $\mathrm{VIII}^{\mathrm{e}}$ siècle. Il comporte en outre un nombre minimum de 46 individus de LRA 5/6 produites localement dans le Mariout, une amphore de facture alluviale, ainsi que les derniers témoins du commerce byzantin méditerranéen: une amphore LRA 4 de Gaza et 3 amphores LRA 1 de Cilicie et de Chypre. Quelques individus appartenant au répertoire culinaire ont aussi été relevés : un manchon de poêle, une marmite à bord biseauté, une cocotte à grandes anses verticales et un couvercle à tenon interne de préhension sont 
tous de facture locale, datés $\mathrm{du} \mathrm{VII}^{\mathrm{e}}$ et du début du $\mathrm{VIII}^{\mathrm{e}}$ siècle. Cet entrepôt, probablement destiné à stocker les amphores vinaires du Mariout et d'Abou Mina, illustre bien le dynamisme économique de la région au tournant de l'Islam. La multitude des types amphoriques représentés illustre aussi le nombre d'ateliers dans la région.

\subsubsection{Secteur $13:$ les thermes}

Les sondages menés en 2019 ont permis de mieux préciser les contextes d'installation du complexe dans le quartier de la ville moyenne de Taposiris. Si les niveaux d'abandon $\mathrm{du}$ bâtiment sont toujours datés à partir du deuxième quart du $\mathrm{VII}^{\mathrm{e}}$ siècle, et caractérisés par les mêmes marqueurs céramiques, en particulier de la céramique du monastère d'Abou Mina et des amphores vinaires LRA 5/6 de facture locale, aucun contexte étendu n'avait jusque-là permis de comprendre sur quoi reposait le complexe fondé au milieu du v viècle (sondage 2015).

\subsubsection{Le contexte 13562 (fouille 2018) : le secteur de service}

L'US 13562 ayant été fouillée en 2018 lors des derniers jours de la mission, son analyse céramologique a été menée en 2019. Ce contexte archéologique provient des parties de service, à l'arrière du complexe balnéaire, et correspond à un niveau coupé par l'installation de ce dernier. Composé d'un minimum de 48 individus bien conservés, il est à dater de l'époque romaine impériale. Il comprend en effet, en plus des $\mathrm{AE}$ alluviales, trois amphores de Tripolitaine et une amphore du type de Forlimpopoli. La vaisselle commune se compose de patina, lopades ainsi que de supports de jarre/ braseros de facture souvent locale du Mariout, du Delta ou de ses franges. On peut

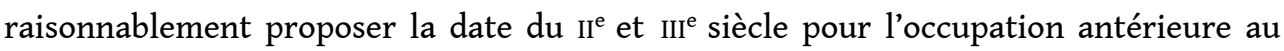
complexe thermal de Taposiris.

\subsubsection{Les boutiques au sud du complexe thermal}

Les niveaux d'installation des structures identifiées comme des boutiques et fouillées lors de la campagne 2019 confirment cette datation de l'époque impériale : les US qui correspondent en partie aux fondations des boutiques fournissent un matériel similaire à celui de l'US 13562 .

\subsubsection{L'espace public à l'est du complexe : le sondage sous la rue}

Un sondage profond a été mené dans l'espace public situé à l'est du complexe sous le niveau de la rue byzantine: il a mis en évidence presque immédiatement sous ses niveaux associés une large fosse comprenant du matériel gréco-romain. Ce dépotoir comporte du matériel ptolémaïque caractéristique de la région et datable des $\mathrm{III}^{\mathrm{e}}$ et $\mathrm{II}^{\mathrm{e}}$ siècles av. J.-C., mais aussi quelques amphores remontant au début du $\mathrm{I}^{\mathrm{er}}$ siècle av. J.C. Le matériel le plus tardif consiste en une amphore de Tripolitaine, probablement datée entre la moitié du $\mathrm{I}^{\mathrm{er}}$ siècle av. J.-C. et le milieu du $\mathrm{I}^{\mathrm{er}}$ siècle apr. J.-C.

\subsection{Céramique de Rahim}

Julie Marchand 
Deux sondages ont été ouverts à Rahim, sur deux petites éminences du site. La prospection de surface qui a été menée confirme les observations préliminaires menées par R. Tomber et R. Thomas entre 2004 et $2008^{2}$ : céramiques ptolémaïque, romaine et byzantine sont bien présentes en surface. Toutefois, la fouille n'a permis de remettre en contexte que deux phases, ptolémaïque (sondage du kôm 2) et byzantine (sondage du kôm 1).

\subsubsection{Le kôm 1 : le complexe balnéaire byzantin}

Sur la première éminence, la prospection de surface présente un matériel homogène daté de la fin de l'Antiquité. Sont ainsi présentes les grandes séries d'amphores vinaires caractéristiques du commerce en Méditerranée, à savoir les LRA 1 et les LRA 4, mais aussi les productions, également vinaires, locales, produites sur la frange sud du Mariout probablement, les LRA 5/6 du Mariout et d'Abou Mina. Enfin, on a collecté une quantité non négligeable de pots de saqiah: différents, dans les types et dans les fabriques, de ceux qui ont été identifiés à Taposiris, ils témoignent de l'intense activité potière sur les berges du lac Mariout durant l'Antiquité tardive.

L'ouverture d'un petit sondage dans le complexe balnéaire confirme la prospection de surface dans le secteur: le complexe a été abandonné dans le courant du $\mathrm{VII}^{\mathrm{e}}$ siècle, voire au début du VIII ${ }^{e}$ siècle. Une grande vasque en marbre blanc, ainsi que quelques fragments d'opus sectile de pierres dures colorées, assignent une certaine importance et un certain rang à ce complexe balnéaire, qui aurait pu appartenir à une villa, peut-être un de ces établissements caractéristiques de la Maréotide qui regroupent une villa, un pressoir (pour le vin) et un atelier d'amphores (et de saqiah?)3.

\subsubsection{Le kôm 2 : le secteur ptolémaïque}

Dans le sondage ouvert par la mission, seule une US très profonde dans le bâtiment a donné de la céramique. L'assemblage donne quantité de céramique importée (de Cnide sans doute et de Rhodes, d'après le timbre), ainsi que quelques conteneurs locaux, qui imitent les formes égéennes. On notera aussi un conteneur égyptien, une siga des oasis occidentales. Enfin, un peu de céramique culinaire locale a été enregistrée. La structure, qui pourrait être un entrepôt d'époque ptolémaïque, pourrait donc avoir été occupée autour du II siècle av. J.-C.

\subsection{Restauration du mobilier métallique et des monnaies}

Mahmoud Samir

En mai 2019, Mahmoud Samir, restaurateur spécialisé dans le traitement des objets en métal, assisté par Mohamed Khamis Ragab, restaurateur au MoA, a achevé le nettoyage des monnaies entreposées au magasin de Shallalat. 220 monnaies ont été nettoyées cette année; elles ont ensuite été identifiées par T. Faucher (cf. infra), photographiées et enregistrées dans une base de données. Mahmoud Samir a également restauré 27 objets en bronze entreposés à Shallalat, dont trois bols en bronze saïte mis au jour à Plinthine et une bague à chaton trouvée à Taposiris. 


\section{5. Étude des monnaies}

Thomas Faucher

Depuis 1998, de très nombreuses monnaies ont été mises au jour sur le site de Taposiris (mais très peu à Plinthine). Elles ne sont pas toutes dans un état satisfaisant de conservation qui permettrait leur étude. Ainsi, sur 351 monnaies restaurées avant 2019 et que nous avons pu étudier cette année, seules 251 pièces sont lisibles (ce qui est toutefois un bon nombre si l'on considère d'autres sites comme Alexandrie). Malgré tout, cette année, 324 monnaies nouvelles ont été enregistrées, ce qui porte à 566 le nombre total de monnaies étudiées par nos soins. L'étude des monnaies s'est déroulée cette année à la fois dans le magasin de Shallalat et sur le site du 6 au 13 mai.

Il faut souligner ici que le matériel numismatique des différents sites fouillés par la mission (ville haute et basse de Taposiris, Plinthine, Qoseir, etc.) donne un point d'attaque tout à fait exceptionnel pour l'étude de la circulation monétaire dans la région. Mais son analyse offre des perspectives encore plus larges à l'échelle du pays, et ce pour différentes périodes d'occupation du site (ptolémaïque, romaine et byzantine). Seule la Basse Époque manque encore à ce tableau puisque les dernières fouilles n'ont pas encore mis au jour de monnaies du ve et des deux premiers tiers du Iv siècle av. J.C., alors qu'on pourrait légitimement en espérer. Quoi qu'il en soit, ce corpus est conséquent. Il se situe bien sûr en dessous des monnaies récoltées dans les fouilles du CEAlex pour Alexandrie (env. 3500 publiées à ce jour) ou dans les fouilles de Karanis ou de Tell Athrib mais est à peu près équivalent à celui des fouilles voisines du CEAlex sur la presqu'île de Maréa. Il en résulte un échantillon qu'on peut penser représentatif de la circulation monétaire aux périodes ptolémaïque, romaine et byzantine.

\begin{tabular}{|l|l|}
\hline Période & Nombre de monnaies \\
\hline Pré-ptolémaïque & 1 \\
\hline Ptolémaïque & 170 \\
\hline Haut Empire romain & 108 \\
\hline Bas Empire romain & 139 \\
\hline Byzantine & 100 \\
\hline Islamique & 22 \\
\hline Moderne & 1 \\
\hline Non identifiée & 25 \\
\hline
\end{tabular}

\section{Publications, valorisation, travaux universitaires}

\subsection{Publications}

\subsubsection{Monographie}

Après l'achèvement du dossier concernant la nécropole hellénistique (à paraître aux presses de l'Ifao), M.-F. Boussac a lancé la publication de la ville basse de Taposiris (secteurs 1, 2, 3, 4, 8, 9, 16, 17, 18, 19). Cette monographie concerne l'ensemble de la 
zone lacustre, dont la levée artificielle d'orientation est-ouest et la jetée nord-sud qui ferme le bassin à l'est. La nature et l'impact des modifications et des évolutions du contexte environnemental font qu'une partie importante de cette monographie sera consacrée à des études géomorphologiques (M. Crépy) portant sur l'histoire et la navigabilité du lac aux différentes époques, et à des études géophysiques (C. Benech), à côté des analyses archéologiques (M. El Amouri, J. Le Bomin, M.-C. Petitpa, V. Pichot, H. Silhouette). Les études de matériel sont faites par C. Harlaut, J. Marchand, S. Marquié, G. Cankardeş-şenol, A. Simony.

Une demande de financement à la fondation Shelby-White a été déposée en janvier 2020 pour faciliter la préparation et la réalisation de la publication du port, sous format papier, mais aussi sous la forme d'un SIG interactif.

\subsubsection{Articles scientifiques}

- Joachim Le Bomin, Julie Marchand, Matthieu Vanpeene, « Se baigner à l'aube de la conquête arabe. La fouille des thermes byzantins de Taposiris Magna », BIFAO 119, 2019, p. 181-224.

- Marie-Françoise Boussac, Bérangère Redon, «The Mareotis Area: Integration of a Marginal Territory into Egypt through the Wine Production ", in Katherine Blouin (éd.), Imperial Landscapes: Empires, Societies, and Environments in the Ancient to Modern Nile Delta (à paraitre).

- Marie-Françoise Boussac, Bérangère Redon, «Définition mouvante et logique d'appropriation des confins nord-ouest de l'Égypte du Nouvel Empire à l'époque romaine. À propos des découvertes archéologiques récentes à Plinthine et Taposiris Magna ", in Marie-Françoise Boussac et al. (éd.), Marges occidentales de l'Égypte. Actes du colloque du Caire, presses de l'Ifao (à paraître).

- Joachim Le Bomin, Julie Marchand, Aude Simony, « Long and Middle Distance Trade in the Occidental Delta during Late Antiquity: The Taposiris Magna and Kom Abu Billu Examples », in Valentina Caminneci, Maria Concetta Parello, Maria Serena Rizzo (éd.), LRCW 6 6th International Conference on Late Roman Coarse Wares, Cooking Wares and Amphorae in the Mediterranean: Archaeology and Archaeometry Land and Sea: Pottery Routes (à paraître).

-Joachim Le Bomin, Julie Marchand, «Saqia Pots from Taposiris Magna », in Valentina Caminneci, Maria Concetta Parello, Maria Serena Rizzo (éd.), LRCW 6 - 6th International Conference on Late Roman Coarse Wares, Cooking Wares and Amphorae in the Mediterranean: Archaeology and Archaeometry Land and Sea: Pottery Routes (à paraître).

\subsubsection{Articles de vulgarisation}

- Bérangère Redon, Sylvain Dhennin, «Plinthine et Térénouthis. Nouvelles découvertes archéologiques dans la marge occidentale de l'Égypte ", Archéologia, hors-série 27, 2019, p. 34-39.

- Bérangère Redon, «An Egyptian Grand Cru: the Wine Production of Plinthine (Mareotis Area), from the New Kingdom to the Ptolemaic Period », EgArch 55, 2019, p. 28-33.

\subsection{Conférences, colloques}

- Maël Crépy, Marie-Françoise Boussac, Joachim Le Bomin, Julie Marchand, MarieChristine Petitpa, « Dater les aménagements lacustres et les structures hydrauliques de Taposiris Magna (II ${ }^{\mathrm{e}}$ s. av. J.-C. - VIII ${ }^{\mathrm{e}}$ s. apr. J.-C.) : approche interdisciplinaire et enjeux socio-environnementaux », journées d'études "Datation des structures hydrauliques: enjeux, questions et méthodes », Lyon, 25 mars 2019. 
- Mennat-Allah El Dorry, Charlène Bouchaud, Clémence Pagnoux, Bérangère Redon, Marie-Françoise Boussac, Rim Saleh, Laurent Bouby, Vincent Bonhomme, Sarah Ivorra, Jean-Frédéric Terral, "Agriculture and Viticulture in the New Kingdom-Antique Egyptian Delta", 18th Conference of the International Workgroup for Palaeoethnobotany, Lecce, 3-8 juin 2019.

- Julie Marchand, Chokri Touihri, "Production, diffusion et consommation de la céramique entre Ifriqiya et le Delta égyptien occidental entre le $7^{\mathrm{e}}$ et le $9^{\mathrm{e}}$ siècle ", colloque "La diffusion de la céramique islamique de Tunisie en Méditerranée », Centre national de la céramique de Tunis, 5 septembre 2019.

- Maël Crépy, Marie-Françoise Boussac, Joachim Le Bomin, Julie Marchand, « Taposiris and Its Harbour: New Data on its Chronology and Layout during Antiquity (from Ptolemaic to Medieval Period) », First Symposium of the Workgroup Egyptian Riverine Harbours, Ifao, Le Caire, 15-18 septembre 2019.

- Joachim Le Bomin, Julie Marchand, Paul Reynolds, « Proto-LRA 1 Amphora Found at Taposiris Magna (Egypt): Some Elements of a Long-lasting Trade? », colloque LRCW 7, Valence, 15 octobre 2019.

- Maël Crépy, "When Sediment Isn't Sufficient: Multidisciplinar and Multiscalar Approaches of the Evolution of the Mareotis Lake(s) during the Late Holocene ", Workshop Geoarchaeology in the Nile Delta, Universität Würzburg, 29-30 novembre 2019.

- Julie Marchand, « Production, diffusion et consommation de la vaisselle de céramique et de pierre en Égypte, entre le $7^{\mathrm{e}}$ et le $9^{\mathrm{e}}$ siècle ", séminaire de recherche, université Paris 1 Panthéon-Sorbonne, 12 novembre 2019.

- Mennat-Allah El Dorry, Charlène Bouchaud, «Archaeobotany of the Egyptian Delta: State of Research ", International Conference Alexandria the Cosmopolis: A Global Perspective, CEAlex, Universiteit Leiden, Alexandrie, 2-5 décembre 2019.

\subsection{Travaux universitaires}

Clémence Pagnoux a été nommée membre scientifique de l'École française d'Athènes à la rentrée 2019. Ses travaux porteront sur les cultures arbustives dans l'Antiquité et le cas de Plinthine constituera l'un de ses principaux dossiers de terrain et d'expérimentation, sur le thème de la vigne.

61 Louis Dautais a obtenu l'allocation doctorale de l'Ifao pour effectuer ses travaux de thèse à partir de septembre 2019 sur le sujet «L'Égypte et le monde égéen (XVII ${ }^{\mathrm{e}}$-XII ${ }^{\mathrm{e}} \mathrm{s}$. av.n. è.) : une approche globale et diachronique de leurs interactions" (dir. M. Gabolde, université Paul-Valéry Montpellier 3 et C. Langhor, UCLouvain).

Maël Crépy a obtenu du CNRS un nouveau CDD de 6 mois dans le cadre du projet ERC de B. Redon (2 $2^{\mathrm{e}}$ semestre 2019).

63 Julie Marchand a obtenu du CNRS un CDD de 11 mois dans le cadre du projet H2020 PREVISION (Prediction and Visual Intelligence for Security Information) de S. Dhennin.

La thèse de Joachim Le Bomin, soutenue le 12 octobre 2018 à l'université de Paris 1 Panthéon-Sorbonne, sous la direction de Michel Kaplan et de Dominique Pieri, intitulée De la Méditerranée vers l'Atlantique: les importations de céramiques méditerranéennes dans l'ouest des Gaules durant l'Antiquité tardive (du milieu du III au début du VII ${ }^{e}$ s. de n.è., a été sélectionnée pour concourir aux prix de la Chancellerie des universités de Paris 2019. 


\section{NOTES}

1. Pour davantage d'informations sur la mission, on consultera le carnet de recherche de la mission : www.taposiris.hypotheses.org (dernière consultation le 8 avril 2020).

2. Site 210-211 dans Lucy Blue, Emad Khalil, A Multidisciplinary Approach to Alexandria's Economic Past: The Lake Mareotis Research Project, BAR-IS 2285, Oxford, Archaeopress, 2011, p. 171-174.

3. Jean-Yves Empereur, "La production viticole en Égypte», in Jean-Yves Empereur, Marie-Claire Amouretti, Jean-Pierre Brun (éd.), La production du vin et de l'huile en Méditerranée, BCH Suppl. 26, 1993, p. 46.

\section{INDEX}

Année de l'opération : 2019

sujets https://ark.frantiq.fr/ark:/26678/pcrtihlJWfvH4v, https://ark.frantiq.fr/ark:/26678/ pcrtYhYMiLwDUr, https://ark.frantiq.fr/ark:/26678/pcrtWegewfItfX, https://ark.frantiq.fr/ ark:/26678/pcrte0Wtwmldeh, https://ark.frantiq.fr/ark:/26678/pcrtIdEjFMFAod, https:// ark.frantiq.fr/ark:/26678/pcrtVemT606YVG, https://ark.frantiq.fr/ark:/26678/pcrtItMnfHySi0, https://ark.frantiq.fr/ark:/26678/crtzvDUrgaTOx, https://ark.frantiq.fr/ark:/26678/ pcrtPe1qxjEkcR

anthroponymes https://ark.frantiq.fr/ark:/26678/pcrt5XAzjOUWxy

Thèmes : IFAO

\section{AUTEURS}

\section{BÉRANGÈRE REDON}

Historienne, archéologue, CNRS, UMR 5189 HiSoMA

\section{SYLVAIN DHENNIN}

Archéologue et égyptologue, CNRS, UMR 5189

\section{CLAIRE SOMAGLINO}

Archéologue et égyptologue, Sorbonne Université / UMR 8167

\section{MATTHIEU VANPEENE}

Doctorant, architecte, Ifao / EPHE-PSL

LORENZO MEDINI

Archéologue et égyptologue, Ifao 


\section{LOUIS DAUTAIS}

Doctorant en égyptologie, université Paul-Valéry Montpellier 3

\section{SÉVERINE MARCHI}

Archéologue, CNRS, UMR 8167, équipe Mondes pharaoniques

RIM SALEH

Doctorante en archéologie, université Lumière Lyon 2

JOACHIM LE BOMIN

Archéologue, université Paris 1 Panthéon-Sorbonne

JULIE MARCHAND

Céramologue, CNRS, UMR 5189

CHARLÈNE BOUCHAUD

Archéobotaniste, CNRS-MNHN

MENNAT-ALLAH EL DORRY

Archéobotaniste, Ifao / PCMA / MoA

CLÉMENCE PAGNOUX

Archéobotaniste, EfA

ROBERT RYNDZIEWICZ

Géophysicien, PCMA

TOMASZ HERBICH

Géophysicien, PCMA

MAËL CRÉPY

Géographe et géomorphologue, CNRS, UMR 5189

\section{ALEXANDRE RABOT}

Spécialiste de SIG, UMR 5189 / université Lumière Lyon 2

\section{PAUL FRANÇOIS}

Doctorant, architecte et ingénieur, université de Nantes

\section{CLEMENTINA CAPUTO}

Céramologue, Ruprecht-Karls-Universität Heidelberg

\section{MIKAËL PESENTI}

Céramologue, université d'Aix-Marseille

MAHMOUD SAMIR

Restaurateur indépendant 


\section{THOMAS FAUCHER}

Numismate, CNRS, Iramat-CEB 